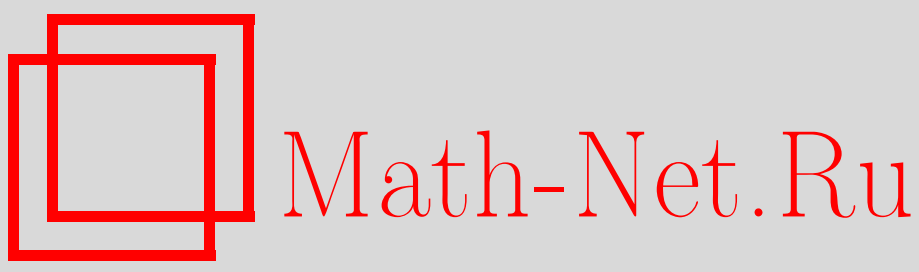

C. Е. Пастухова, Усреднение задач теории упругости на периодических составных структурах, Матем. сб., 2005, том 196, номер 7, 101-142

DOI: https://doi.org/10.4213/sm1402

Использование Общероссийского математического портала Math-Net.Ru подразумевает, что вы прочитали и согласны с пользовательским соглашением

http: //www. mathnet.ru/rus/agreement

Параметры загрузки:

IP : 35.173 .137 .237

26 апреля 2023 г., 15:41:10 
УДК 517.9

\section{С.Е. Пастухова}

\section{Усреднение задач теории упругости на периодических составных структурах}

Рассмотрены задачи теории упругости на плоской плите, армированной периодической тонкой сеткой, или в трехмерном теле, армированном периодическим тонким ящичньм каркасом. Составная среда зависит от двух стремящихся к нулю параметров, контролирующих ячейку периодичности и толщину армирующей структуры. Параметры могут быть как зависимьми, так и независимьми.

Для указанных задач с помошью метода В.В. Жикова "двухмасштабной сходимости с переменной мерой" получен принцип усреднения: решение исходной задачи сходится в определенном смысле к решению усредненной (или предельной) задачи. Последняя имеет классический вид. Из операторной формы принципа усреднения на основе установленного принципа компактности в $L^{2}$-пространстве на составной структуре выводится также сходимость по Хаусдорфу спектра исходной задачи к спектру предельной задачи.

Библиография: 28 названий.

\section{$\S 1$. Введение}

Обозначим через $F$ сетку на плоскости, составленную из всевозможных прямых $x_{i}=a$, где $a$ - любое целое число, $i=1,2$. Каждая такая прямая является продольной осью полосы, ширина которой равна $h \in(0,1 / 2)$. Объединение всех этих полос задает тонкую сетку $F^{h}, h>0$. Квадрат $Y=[-1 / 2,1 / 2)^{2}$ является ячейкой периодичности, $F \cap Y=I_{1} \cup I_{2}$, где $I_{1}, I_{2}$ - единичные интервалы на соответствующих осях. Условимся называть сетку $F=F^{0}$ толщины $h=0$ сингулярной.

Введем на сетках $F^{h}$ естественные меры: $\lambda^{h}, h>0,-$ мера, сосредоточенная на тонкой сетке $F^{h}$ и пропорциональная там плоской мере Лебега, $\lambda=\lambda^{0}$ - мера, сосредоточенная на сингулярной сетке $F$ и пропорциональная там линейной мере Лебега. Меры нормированы условием $\int_{Y} d \lambda^{h}=\int_{Y} d \lambda=1$. По построению

$$
d \lambda= \begin{cases}\frac{1}{2} d x_{1} & \text { на } I_{1}, \\ \frac{1}{2} d x_{2} & \text { на } I_{2} .\end{cases}
$$

Имеет место сходимость мер $\lambda^{h} \rightarrow \lambda$ при $h \rightarrow 0$, что означает

$$
\int_{Y} \varphi d \lambda^{h} \rightarrow \int_{Y} \varphi d \lambda \quad \forall \varphi \in C_{\mathrm{per}}^{\infty}(Y)
$$

Работа выполнена при частичной финансовой поддержке Российского фонда фундаментальных исследований (грант № 05-01-00621). 
Рассмотрим составные 1-периодические меры $\mu^{h}, \mu=\mu^{0}$, такие, что

$$
d \mu^{h}=\frac{1}{2} d x+\frac{1}{2} d \lambda^{h}, \quad d \mu=\frac{1}{2} d x+\frac{1}{2} d \lambda, \quad \mu^{h} \rightarrow \mu,
$$

где $d x$ - плоская мера Лебега.

Гомотетическое сжатие плоскости в $\varepsilon^{-1}$ раз переводит сетки $F^{h}$ и $F$ в $F_{\varepsilon}^{h}$ и $F_{\varepsilon}$ соответственно, при этом возникают масштабированные меры $\mu_{\varepsilon}^{h}, \mu_{\varepsilon}$, для которых

$$
\mu_{\varepsilon}^{h}(B)=\varepsilon^{2} \mu^{h}\left(\varepsilon^{-1} B\right), \quad \mu_{\varepsilon}(B)=\varepsilon^{2} \mu\left(\varepsilon^{-1} B\right),
$$

где $B$ - любое борелево множество на плоскости. Очевидно, что

$$
d \mu_{\varepsilon}^{h}=\frac{1}{2} d x+\frac{1}{2} d \lambda_{\varepsilon}^{h}, \quad d \mu_{\varepsilon}=\frac{1}{2} d x+\frac{1}{2} d \lambda_{\varepsilon},
$$

где $\varepsilon$-периодические меры $\lambda_{\varepsilon}^{h}, \lambda_{\varepsilon}$ сосредоточены на сетках $F_{\varepsilon}^{h}$ и $F_{\varepsilon}$. Поскольку $\mu_{\varepsilon}^{h}(\varepsilon Y)=\mu_{\varepsilon}(\varepsilon Y)=\varepsilon^{2}$, то $d \mu_{\varepsilon}^{h} \rightarrow d x, d \mu_{\varepsilon} \rightarrow d x$ при $\varepsilon \rightarrow 0$, т.е.

$$
\int_{\mathbb{R}^{2}} \varphi d \mu_{\varepsilon}^{h} \rightarrow \int_{\mathbb{R}^{2}} \varphi d x \quad \forall \varphi \in C_{0}^{\infty}\left(\mathbb{R}^{2}\right)
$$

где $h \geqslant 0, \mu_{\varepsilon}^{0}=\mu_{\varepsilon}$.

Тензор упругости $A=\left\{a_{i j s p}\right\}$ подчинен обычньмм условиям симметрии $a_{i j s p}=$ $a_{s p i j}=a_{j i s p}$. Действие тензора $A$ на симметрическую матрицу $\xi$ есть симметрическая матрица $A \xi=\left\{a_{i j s p} \xi_{s p}\right\}$. Предполагаем $A$ положительно определенным: $A \xi \cdot \xi \geqslant c_{0} \xi \cdot \xi, c_{0}>0$. Здесь $\xi \cdot \eta=\xi_{i j} \eta_{i j}-$ скалярное произведение матриц. В изотропном случае

$$
\begin{gathered}
A \xi=k \xi+k_{1}(\operatorname{Tr} \xi) I, \quad k>0, \quad k_{1} \geqslant 0 \\
\xi=\left(\begin{array}{ll}
\xi_{11} & \xi_{12} \\
\xi_{12} & \xi_{22}
\end{array}\right), \quad \operatorname{Tr} \xi=\xi_{11}+\xi_{22}, \quad I=\left(\begin{array}{ll}
1 & 0 \\
0 & 1
\end{array}\right) .
\end{gathered}
$$

Пусть $\Omega$ - ограниченная липшицева область в $\mathbb{R}^{2}, f \in C^{\infty}(\bar{\Omega})^{2}, e(u)=\frac{1}{2}(\nabla u+$ $\left.(\nabla u)^{T}\right), u=\left(u_{1}, u_{2}\right)$. Рассмотрим вариационные задачи на отыскание

$$
\begin{aligned}
J^{\varepsilon, h} & =\inf _{u \in C_{0}^{\infty}(\Omega)^{2}} \int_{\Omega}(A e(u) \cdot e(u)-2 f \cdot u) d \mu_{\varepsilon}^{h}, \\
J^{\varepsilon} & =\inf _{u \in C_{0}^{\infty}(\Omega)^{2}} \int_{\Omega}(A e(u) \cdot e(u)-2 f \cdot u) d \mu_{\varepsilon} .
\end{aligned}
$$

В задачах $(1.3),(1.4)$ можно заменить инфимум по $C_{0}^{\infty}(\Omega)^{2}$ на минимум по соответствуюшему соболевскому пространству (см. $\S \S 4,6)$, который достигается на единственных элементах $u^{\varepsilon, h}$ и $u^{\varepsilon}$, называемых далее минимизантами. Это следует из равномерного (по $\varepsilon$ и $h \geqslant 0)$ неравенства Корна (доказательство см. в $\S 9$ )

$$
\int_{\Omega}|u|^{2} d \mu_{\varepsilon}^{h} \leqslant C \int_{\Omega}|e(u)|^{2} d x, \quad u \in C_{0}^{\infty}(\Omega)^{2}, \quad C=\operatorname{const}(\Omega, F) .
$$


Общая теория усреднения вариационных задач [1], [2] дает следующий результат: при $h=$ const $\geqslant 0$ и $\varepsilon \rightarrow 0$ энергии $J^{\varepsilon, h}, J^{\varepsilon}$ и минимизанты $u^{\varepsilon, h}, u^{\varepsilon}$ сходятся к энергиям $J^{h}, J$ и минимизантам $u^{h}, u$ вариационных задач на отыскание минимума энергии

$$
\begin{aligned}
J^{h} & =\min _{u \in H_{0}^{1}(\Omega)^{2}} \int_{\Omega}\left(A_{h}^{\mathrm{hom}} e(u) \cdot e(u)-2 f \cdot u\right) d x, \\
J & =\min _{u \in H_{0}^{1}(\Omega)^{2}} \int_{\Omega}\left(A^{\mathrm{hom}} e(u) \cdot e(u)-2 f \cdot u\right) d x .
\end{aligned}
$$

Усредненные тензоры $A_{h}^{\text {hom }}$ и $A^{\text {hom }}$ задаются формулами

$$
\begin{aligned}
& A_{h}^{\text {hom }} \xi \cdot \xi=\inf _{u \in C_{\text {per }}^{\infty}(Y)^{2}} \int_{Y} A(\xi+e(u)) \cdot(\xi+e(u)) d \mu^{h}, \\
& A^{\text {hom }} \xi \xi=\inf _{u \in C_{\text {per }}^{\infty}(Y)^{2}} \int_{Y} A(\xi+e(u)) \cdot(\xi+e(u)) d \mu .
\end{aligned}
$$

Классический характер усредненных задач $(1.6),(1.7)$ определяется тем, что лишь постоянные векторы являются жесткими периодическими перемешениями в пространствах $L^{2}\left(Y, d \mu^{h}\right)^{2}$ и $L^{2}(Y, d \mu)^{2}$.

В связи с задачами (1.3), (1.4) возникает следуюший круг вопросов.

$1^{\circ}$. Задача (1.3) зависит через меру от второго параметра $h$. Актуален вопрос о предельном переходе по параметру $h \rightarrow 0$ до и после усреднения по $\varepsilon$ : действительно ли имеют место ожидаемые в соответствии с обозначениями равенства

$$
\lim _{h \rightarrow 0} J^{\varepsilon, h}=J^{\varepsilon} \quad \text { и } \quad \lim _{h \rightarrow 0} J^{h}=J
$$

которые делают диаграмму предельных переходов

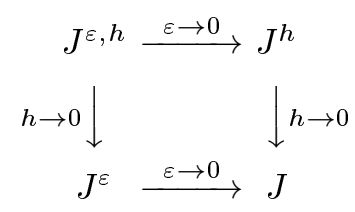

замкнутой и коммутативной. Утвердительный ответ на этот вопрос дается в теореме 6.1.

$2^{\circ}$. До сих пор параметры $\varepsilon, h$ считались независимыми. Изучим усреднение задачи (1.3) в том случае, когда $h=h(\varepsilon) \rightarrow 0$ при $\varepsilon \rightarrow 0$, т.е. толщина сетки $F^{h}$, равная $h(\varepsilon)$, стремится к нулю при $\varepsilon \rightarrow 0$, так что $F^{h}$ переходит в сингулярную сетку $F$. Асимптотически сингулярные структуры принято называть тонкими [3].

Если в задаче (1.3) вместо меры $\mu_{\varepsilon}^{h}$ взять меру $\lambda_{\varepsilon}^{h}$ (т.е. рассматривать вариационную задачу на тонкой сетке), усреднение будет зависеть от того, какова величина $\theta=\lim _{\varepsilon \rightarrow 0} h(\varepsilon) / \varepsilon: \theta=0, \theta \in(0, \infty), \theta=\infty[3]-[5]$. Вид усредненной задачи и тип сходимости минимизантов $u^{\varepsilon, h}$ различаются для трех перечисленных случаев. 
Причину этого масштабного эффекта вскрывает неравенство Корна на $\varepsilon$-периодических тонких сетках $F_{\varepsilon}^{h}[5],[6]$ :

$$
\int|u|^{2} d \lambda_{\varepsilon}^{h} \leqslant c\left(\left(\frac{\varepsilon}{h}\right)^{2}+1\right) \int|e(u)|^{2} d \lambda_{\varepsilon}^{h}, \quad u \in C_{0}^{\infty}(\Omega)^{2},
$$

где $c$ - константа, зависяшая лишь от диаметра $\Omega$, а порядки, с которьми входят в правую часть параметры $\varepsilon$ и $h$, точны. Видим, что константа $C=c\left((\varepsilon / h)^{2}+1\right)$ в правой части (1.11) может расти к бесконечности. Ограниченность аналогичной константы в неравенстве Корна (1.5) исключает масштабный эффект при усреднении задачи (1.3) с составной мерой $\mu_{\varepsilon}^{h(\varepsilon)}, h=h(\varepsilon) \rightarrow 0$.

Кроме того, для составной меры $\mu$, в отличие от меры $\lambda$, множество периодических жестких перемешений исчерпывается постоянными векторами (см. §5). Тогда предельная задача для составных структур не может быть “дву хмасштабной”, как для тонких сеток.

Отмеченные выше факторы: отсутствие масштабного эффекта в неравенстве Корна и тривиальность множества периодических жестких перемешений, определяют классический характер принципа усреднения на составных структурах с тонкой составляюшей. При любой $h(\varepsilon) \rightarrow 0$ имеет место (см. теорему 6.2) сильная сходимость в пространстве $L^{2}(\Omega, d x)^{2}$ решений исходной задачи (1.3) к решению обычной усредненной задачи (1.7), дополненная сходимостью энергий:

$$
u^{\varepsilon, h} \rightarrow u \text { в } L^{2}(\Omega)^{2}, \quad J^{\varepsilon, h} \rightarrow J .
$$

Вывод соотношений (1.12) использует двухмасштабную сходимость с переменной мерой $\mu^{h}$, которая изучается в $\S 7$. Для того чтобы эта сходимость обладала необходимьми для техники усреднения свойствами, требуется вьполнение так называемых аппроксимативных условий для меры $\mu^{h}$. Их проверка приведена в $\S 5$. Первоначально принцип усреднения дается в терминах сильной сходимости в переменном пространстве $L^{2}\left(\Omega, d \mu_{\varepsilon}^{h}\right)$, а затем благодаря свойствам этой сходимости (см. 99$)$ делается переход к обычному для классического усреднения соотношению (1.12) 1 .

Принцип усреднения допускает операторную форму сильной резольвентной сходимости в $L^{2}\left(\Omega, d \mu_{\varepsilon}^{h}\right)^{2}$. В этом пространстве справедлив также принцип компактности (см. §9). Отсюда (см. §10) выводится сходимость по Хаусдорфу спектра исходной задачи к спектру предельной задачи, а также более точная сходимость собственных чисел и собственных векторов оператора исходной задачи. При этом рассмотрена обшая схема (включаюшая и некоторые другие задачи усреднения) операторов, действующих в переменном гильбертовом пространстве.

$3^{\circ}$. Рассматривая уравнение Эйлера для задачи (1.4), мы приходим (см. §4) к классической его форме, которая представляет собой смешанную задачу с условием Дирихле на внешней границе $\partial \Omega$ и с краевым условием типа Вентцеля на каждом звене сетки $F_{\varepsilon} \cap \Omega$. Аналогично для периодической задачи $(1.8)_{2}$ в классической форме уравнения Эйлера появляется краевое условие типа Вентцеля на каждом звене сетки $F$. Чтобы получить классическую форму уравнения Эйлера для задач $(1.4),(1.8)_{2}$, мы проводим предварительную релаксацию минимизируемых функционалов на сингулярной сетке $F_{\varepsilon}$ или $F$ соответственно. Отметим, 
что релаксация выпуклых функционалов, заданных в соболевских пространствах скалярных функций с произвольной мерой, рассматривалась и другими авторами (см., например, [2], [7], [8]).

Идея релаксации позволяет вычислить для составной структуры усредненный тензор $A^{\text {hom }}$ из $(1.8)_{2}$ при произвольном тензоре упругости $A$. В изотропном случаe (cм. (1.2))

$$
A^{\text {hom }} \xi \cdot \xi=\frac{1}{2} k \xi \cdot \xi+\frac{1}{2} k_{1}(\operatorname{Tr} \xi)^{2}+\frac{1}{4} \widehat{k}\left(\xi_{11}^{2}+\xi_{22}^{2}\right), \quad \widehat{k}=\frac{k\left(k+2 k_{1}\right)}{k+k_{1}} .
$$

Аналогичная методика применялась в [9], [10] при изучении усредненного тензора для периодических сеток.

$4^{\circ}$. Мера $\mu^{h}$ абсолютно непрерывна относительно меры Лебега: $d \mu^{h}=\rho^{h}(x) d x$ и плотность $\rho^{h}(x)$ сильно контрастна при $h \rightarrow 0$ (порядка $O\left(h^{-1}\right)$ на $F^{h}$ и $O(1)$ вне $F^{h}$ ). В этом смысле мера $\mu^{h}$ описывает плоскость, армированную тонкой сеткой. В трехмерном случае с помошью аналогичной меры $\mu^{h}$ можно описать пространство, армированное тонкой ящичной структурой $F^{h}$. Соответствующая сингулярная ящичная структура $F$ составлена из трех семейств плоскостей $x_{i}=a$, где $a$ - любое целое число, $i=1,2,3$. Для таких трехмерных составных мер задачи (1.3), (1.4) имеют аналогичное усреднение, как в плоском случае.

Заметим, что усреднение сильно контрастных композитов в скалярных и векторных задачах изучалось и ранее, например в работах [11; гл. 7], [12]-[15], но при условии, что ни одна из фаз композита не является тонкой или сингулярной структурой.

$5^{\circ}$. В составной структуре на плоскости в качестве армируюшей составляющей $F$ может быть не только квадратная сетка, но и составленный из отрезков периодический связный граф̆ достаточно обшего вида. Соответствующая тонкая армируюшая сетка $F^{h}$ получается из $F$ заменой каждого отрезка на полосу ширины $h$. Составная структура с квадратной сеткой выделена особо, потому что для нее обоснование процедуры усреднения существенно проще, чем в общем случае. А именно проверка необходимых для усреднения аппроксимативных свойств меры $\mu^{h}$ может быть проделана для квадратной сетки $F$ более или менее просто - сведением к случаю так называемых мер сглаживания, для которых выполнение аппроксимативных условий - общий факт, установленный В. В. Жиковым [3]. В общем случае проверка аппроксимативных свойств технична и требует введения дополнительных (не используемых в других разделах) понятий и построений и по этой причине отнесена в самый конец статьи, в $§ 11$.

$6^{\circ}$. Представляют интерес вариационные задачи, аналогичные (1.3), (1.4), когда составная структура наиболее простая, а именно - плита с одиночньм стержнем, сингулярным или тонким. Мы изучаем эти задачи в $\S \S 2,3$, их скалярные аналоги рассматривались в [16]-[18].

\section{§2. Задача на плите с бесконечно тонким стержнем}

Вариационная постановка задачи. На квадрате $\Omega=(0,1) \times(-1 / 2,1 / 2)$ со средней линией $I=(0,1) \times\{0\}$ введем составную меру

$$
\mu=\left.d x\right|_{\Omega}+d \lambda, \quad d \lambda=\left.d x_{1}\right|_{I}
$$


Далее $\mathscr{A}$ - тензор упругости, удовлетворяющий обычным условиям симметрии и положительной определенности. Обозначим через $C_{\Gamma}^{\infty}(\bar{\Omega})$ множество функций из $C^{\infty}(\bar{\Omega})$, равных нулю в окрестности $\Gamma=\{0\} \times(-1 / 2,1 / 2)$.

Нас будет интересовать вариационная задача на отыскание

$$
J=\inf _{u \in C_{\Gamma}^{\infty}(\bar{\Omega})^{2}} \int_{\Omega}(\mathscr{A} e(u) \cdot e(u)-2 G \cdot u) d \mu,
$$

что в более подробной записи означает

$$
J=\inf _{u \in C_{\Gamma}^{\infty}(\bar{\Omega})^{2}}\left(\int_{\Omega} A e(u) \cdot e(u) d x+\int_{\Omega} B e(u) \cdot e(u) d \lambda-2 \int_{\Omega} f \cdot u d x-2 \int_{\Omega} g \cdot u d \lambda\right) .
$$

Здесь тензор упругости $\mathscr{A}$ и функция $G$ представлены парой компонент: $\mathscr{A}=$ $(A, B), G=(f, g)$, одна компонента "действует" на квадрате $\Omega$, а другая - на интервале $I ; G \in L^{2}(\Omega, d \mu)^{2}$, т.е. $f \in L^{2}(\Omega, d x)^{2}, g \in L^{2}(\Omega, d \lambda)^{2}$.

Пусть $W_{\Gamma}$ - замькание множества $\left\{(u, e(u)): u \in C_{\Gamma}^{\infty}(\bar{\Omega})^{2}\right\}$ в $L^{2}(\Omega, d \mu)^{2} \times$ $L^{2}(\Omega, d \mu)^{3}$. Элементами пространства $W_{\Gamma}$ служат пары $(u, v), u$-вектор, $v$-матрица. Матрицу $v$ назьваем симметрическим градиентом (или просто градиентом) вектора $u$ и обозначаем через $e(u)$. Вектор $u$ может иметь много градиентов. Пусть $\mathscr{E}(u)$ - множество всех градиентов функции $u$. Его структура такова:

$$
\mathscr{E}(u)=e(u)+\mathscr{E}(0),
$$

где $e(u)$ - какой-то фиксированный градиент, а $\mathscr{E}(0)$ - множество градиентов нуля. По определению $z \in \mathscr{E}(0)$, если существует последовательность $\varphi^{n} \in C_{\Gamma}^{\infty}(\bar{\Omega})^{2}$ такая, что

$$
\varphi^{n} \rightarrow 0 \text { в } L^{2}(\Omega, d \mu)^{2}, \quad e\left(\varphi^{n}\right) \rightarrow z \text { в } L^{2}(\Omega, d \mu)^{3} .
$$

Нетривиальность градиента нуля $z \not \equiv 0$ проявляется только на $I$, где согласно [3] $\left.z\right|_{I}=\left(\begin{array}{cc}0 & \alpha \\ \alpha & \beta\end{array}\right), \alpha, \beta-$ произвольные функции из $L^{2}(I)$.

Ясно, что $\mathscr{E}(0)$ - замкнутое пространство в $L^{2}(\Omega, d \mu)^{3}$. Определим множество тангенциальных матриц как ортогональное в $L^{2}(\Omega, d \mu)^{3}$ дополнение $\mathscr{E}(0)^{\perp}$.

Из общего результата [3; лемма 5.1] о том, что свойство тангенциальности можно определить поточечно, для нашего случая вытекает

Лемма 2.1. Симметрическая матрица $z \in L^{2}(\Omega, d \mu)^{3}$ тангенциальна $\Longleftrightarrow$ $\left.z\right|_{I}=\left(\begin{array}{cc}\alpha & 0 \\ 0 & 0\end{array}\right), \alpha \in L^{2}(I)$.

Любой градиент $e(u)$ допускает единственное представление в виде суммы градиента нуля и тангенциальной матрицы

$$
e(u)=e^{t}(u)+z, \quad z \in \mathscr{E}(0), \quad e^{t}(u) \perp \mathscr{E}(0) .
$$

Компоненту $e^{t}(u)$ назовем тангенииальным градиентом функции $u$. Структура множества $\mathscr{E}(0)$ определяет структуру тангенциального градиента

$$
e^{t}(u)=e(u) \text { вне } I, \quad e^{t}(u)=\left(\begin{array}{cc}
\frac{\partial u_{1}}{\partial x_{1}} & 0 \\
0 & 0
\end{array}\right) \quad \text { на } I .
$$


Пусть $\mathscr{H}(\Omega, d \mu)=\left\{u:(u, v) \in W_{\Gamma}\right\}$. Можно дать описание этого пространства в терминах обычных соболевских пространств.

Формально следуя определению, элемент из $\mathscr{H}(\Omega, d \mu)$ надо представлять парой вектор-функций $u,\left.u\right|_{I}$, определенных в области $\Omega$ и на интервале $I$ соответственно, таких, что найдется $u^{\delta} \in C_{\Gamma}^{\infty}(\bar{\Omega})^{2}$ :

$$
\begin{aligned}
& \int_{\Omega}\left(\left|u-u^{\delta}\right|^{2}+\left|e(u)-e\left(u^{\delta}\right)\right|^{2}\right) d x \rightarrow 0, \\
& \int_{\Omega}\left(|u|_{I}-\left.u^{\delta}\right|^{2}+\left|e\left(\left.u\right|_{I}\right)-e\left(u^{\delta}\right)\right|^{2}\right) d \lambda \rightarrow 0 .
\end{aligned}
$$

Отсюда, а также из обычного неравенства Корна

$$
\int_{\Omega}|\nabla v|^{2} d x \leqslant C \int_{\Omega}\left(|v|^{2}+|e(v)|^{2}\right) d x, \quad v \in C^{\infty}(\bar{\Omega})^{2}
$$

и неравенства для следа

$$
\int_{I} \varphi\left(x_{1}, 0\right)^{2} d x_{1} \leqslant C \int_{\Omega}\left(\varphi^{2}+|\nabla \varphi|^{2}\right) d x, \quad \varphi \in C^{\infty}(\bar{\Omega})^{2}
$$

получаем свойства вектор-функций $u,\left.u\right|_{I}$ :

$$
\begin{aligned}
& u \in H^{1}(\Omega)^{2}, \\
& \left.u_{1}\right|_{I},\left.u_{2}\right|_{I}-\text { следы функций } u_{1}, u_{2} \in H^{1}(\Omega) \text { на } I, \\
& \left.u_{1}\right|_{I} \in H^{1}(I) .
\end{aligned}
$$

Третье свойство обеспечено соотношениями: $u_{1}^{\delta} \rightarrow u_{1}, \frac{\partial u_{1}^{\delta}}{\partial x_{1}}=e_{11}\left(u^{\delta}\right) \rightarrow e_{11}(u)=$ $\frac{\partial u_{1}}{\partial x_{1}}$ в $L^{2}(I)$, которые следуют из определяющих вектор $u$ сходимостей с учетом второго свойства.

Видим, что последовательность $u^{\delta}$ порождает, на самом деле, одну вектор-функцию $u \in H^{1}(\Omega)^{2}$, у которой след на отрезок $I$ имеет продольную компоненту повышенной гладкости.

Вернемся к задаче (2.2). Ее можно сформулировать как задачу на отыскание минимума энергии:

$$
J=\min _{(u, e(u)) \in W_{\Gamma}} \int_{\Omega}(\mathscr{A} e(u) \cdot e(u)-2 G \cdot u) d \mu
$$

или, в более подробной записи,

$$
J=\min _{(u, e(u)) \in W_{\Gamma}}\left(\int_{\Omega} A e(u) \cdot e(u) d x+\int_{\Omega} B e(u) \cdot e(u) d \lambda-2 \int_{\Omega} f \cdot u d x-2 \int_{\Omega} g \cdot u d \lambda\right) .
$$


Минимизант задачи (2.5) - пара, состоящая из функции $u$ и какого-то ее градиента $e(u)$. Эта пара удовлетворяет интегральному тождеству (обобщенному уравнению Эйлера):

$$
\int_{\Omega} \mathscr{A} e(u) \cdot e(\varphi) d \mu=\int_{\Omega} G \cdot \varphi d \mu \quad \forall \varphi \in C_{\Gamma}^{\infty}(\bar{\Omega})^{2}
$$

или, в более подробной записи,

$\int_{\Omega} A e(u) \cdot e(\varphi) d x+\int_{\Omega} B e(u) \cdot e(\varphi) d \lambda=\int_{\Omega} f \cdot \varphi d x+\int_{\Omega} g \cdot \varphi d \lambda \quad \forall \varphi \in C_{\Gamma}^{\infty}(\bar{\Omega})^{2}$.

Сушествование и единственность решения задачи (2.6) доказывается с помощью следуюшего неравенства Корна:

$$
\int_{\Omega}|u|^{2} d \mu \leqslant c \int_{\Omega}|e(u)|^{2} d \mu, \quad u \in C_{\Gamma}^{\infty}(\bar{\Omega})^{2} .
$$

В более подробной записи (2.7) означает, что

$$
\int_{\Omega}|u|^{2} d x+\int_{I}|u|^{2} d x_{1} \leqslant c\left(\int_{\Omega}|e(u)|^{2} d x+\int_{I}|e(u)|^{2} d x_{1}\right), \quad u \in C_{\Gamma}^{\infty}(\bar{\Omega})^{2},
$$

и потому вытекает из неравенства для следа функции и обычного неравенства Корна

$$
\int_{\Omega}\left(|u|^{2}+|\nabla u|^{2}\right) d x \leqslant c \int_{\Omega}|e(u)|^{2} d x, \quad u \in C_{\Gamma}^{\infty}(\bar{\Omega})^{2} .
$$

В силу (2.7) левая часть тождества (2.6) задает скалярное произведение в пространстве $W_{\Gamma}$, соответствующую норму назовем энергетической. Правая часть (2.6) есть линейный функционал, ограниченный по энергетической норме. Тогда однозначная разрешимость задачи (2.6) следует из теоремы Рисса о представлении линейного функционала в гильбертовом пространстве. При этом единственность решения здесь двоякого рода: одна функция $u$ и один ее градиент $e(u)$ удовлетворяют тождеству (2.6). Заметим, что в энергетической норме участвует лишь вторая компонента пары $(u, e(u)) \in W_{\Gamma}$. Неравенство Корна $(2.7)$ показывает, что по второй компоненте первая определяется однозначно.

Обратим внимание на то, что градиент $e(u)$, участвующий в тождестве $(2.6)$, удовлетворяет условию

$$
\mathscr{A} e(u) \perp \mathscr{E}(0),
$$

которое зависит лиш от тензора $\mathscr{A}$, точнее от его компоненты $\left.\mathscr{A}\right|_{I}=B$. Чтобы убедиться в этом, надо подставить в (2.6) пробные функции $\varphi=\varphi^{n}$ из (2.3) и перейти к пределу при $n \rightarrow \infty$. Соотношение (2.9) означает: $p=\mathscr{A} e(u)$ - тангенциальная матрица, т.е. на отрезке $I p=\left(\begin{array}{cc}p_{11} & 0 \\ 0 & 0\end{array}\right)$. Удобнее иметь дело с таким решением задачи (2.6), когда сам градиент $e(u)$, а не тензор $\mathscr{A} e(u)$, является тангенциальной матрицей, т.е.

$$
e(u)=e^{t}(u)=\left(\begin{array}{cc}
\frac{\partial u_{1}}{\partial x_{1}} & 0 \\
0 & 0
\end{array}\right) \text { на отрезке } I \text {. }
$$

Изменим тензор $\left.\mathscr{A}\right|_{I}=B$ так, чтобы задача $(2.5)$ с измененным тензором имела бы в качестве решения пару $\left(u, e^{t}(u)\right)$ с той же функцией $u$. Эта процедура назьвается релаксацией. 
Тензор релаксации. Пусть $B$ - положительно определенный симметрический оператор, действуюший в пространстве $\mathbb{R}^{3}$ симметрических матриц

$$
\xi=\left(\begin{array}{ll}
\xi_{11} & \xi_{12} \\
\xi_{12} & \xi_{22}
\end{array}\right)
$$

и $\mathscr{E}=\left\{\xi \in \mathbb{R}^{3}: \xi_{11}=0\right\}$.

По определению релаксация $\widehat{B}$ относительно пространства $\mathscr{E}$ задается соотношением

$$
\widehat{B} \xi \cdot \xi=\min _{\zeta \in \mathscr{E}} B(\xi+\zeta) \cdot(\xi+\zeta) .
$$

Минимум в этой вариационной задаче достигается на элементе

$$
\zeta \in \mathscr{E}, \quad B(\xi+\zeta) \perp \mathscr{E} .
$$

Из $(2.11)_{2}$ следует равенство $B(\xi+\zeta)=c \eta, c=$ const, $\eta=\left(\begin{array}{ll}1 & 0 \\ 0 & 0\end{array}\right)$. Поэтому $\xi+\zeta=c B^{-1} \eta, \xi \cdot \eta=c B^{-1} \eta \cdot \eta$ в силу $(2.11)_{1}$ и, значит, $c=\xi \cdot \eta /\left(B^{-1} \eta \cdot \eta\right)$. Отсюда

$$
\widehat{B} \xi \cdot \xi=B(\xi+\zeta) \cdot \xi=c \eta \cdot \xi=\frac{|\xi \cdot \eta|^{2}}{B^{-1} \eta \cdot \eta}=b \xi_{11}^{2}, \quad \text { где } \quad b=\left(B^{-1} \eta \cdot \eta\right)^{-1} .
$$

Для изотропного тензора $B$ (см. (1.2)) несложные вычисления дают значение $b=\widehat{k}=k\left(k+2 k_{1}\right) /\left(k+k_{1}\right)$.

Перечислим некоторые свойства тензора релаксации $\widehat{B}$, вытекающие из определения (2.10):

$$
\begin{gathered}
\operatorname{Ker} \widehat{B}=\mathscr{E}, \\
\mathscr{E}^{\perp}-\text { область значений тензора } \widehat{B}, \\
B \xi=\widehat{B} \xi, \quad \text { если } B \xi \in \mathscr{E}^{\perp} .
\end{gathered}
$$

Релаксация исходной задачи и классическая форма уравнения Эйлеpa. Вернемся к задаче (2.5). В силу представления (2.4) для произвольного градиента $e(u)$

$$
\begin{gathered}
J=\min _{(u, z)}\left(\int_{\Omega} A e(u) \cdot e(u) d x+\int_{\Omega} B\left(e^{t}(u)+z\right) \cdot\left(e^{t}(u)+z\right) d \lambda-2 \int_{\Omega} G \cdot u d \mu\right), \\
u \in \mathscr{H}(u, d \mu), \quad z \in \mathscr{E}(0) .
\end{gathered}
$$

Напомним (см. лемму 2.1), что ортогональность между множеством градиентов нуля и тангенциальными матрицами можно понимать как в смысле $L^{2}(\Omega, d \mu)^{3}$, так поточечно $\mu$-п.в. Тогда, используя определение тензора релаксации $\widehat{B}$ и формулу (2.12), можем найти

$$
\min _{z \in \mathscr{E}(0)} \int_{\Omega} B\left(e^{t}(u)+z\right) \cdot\left(e^{t}(u)+z\right) d \lambda=\int_{\Omega} \widehat{B} e^{t}(u) \cdot e^{t}(u) d \lambda=\int_{I} b\left(\frac{\partial u_{1}}{\partial x_{1}}\right)^{2} d x_{1}
$$


и, значит,

$$
J=\min _{u \in \mathscr{H}(\Omega, d \mu)}\left(\int_{\Omega} A e(u) \cdot e(u) d x+b \int_{I}\left(\frac{\partial u_{1}}{\partial x_{1}}\right)^{2} d x_{1}-2 \int_{\Omega} G \cdot u d \mu\right) .
$$

Тогда тождество Эйлера для минимизанта $u$ имеет вид

$$
\int_{\Omega} A e(u) \cdot e(\varphi) d x+b \int_{I} \frac{\partial u_{1}}{\partial x_{1}} \frac{\partial \varphi_{1}}{\partial x_{1}} d x_{1}=\int_{\Omega} G \cdot \varphi d \mu \quad \forall \varphi \in C_{\Gamma}^{\infty}(\bar{\Omega})^{2}
$$

Отсюда можно получить классическую форму уравнения Эйлера. Для достаточно гладкой функции $u$ по формуле Грина-Бетти имеем равенство

$$
\begin{aligned}
\int_{\Omega} & A e(u) \cdot e(\varphi) d x=\int_{\Omega \backslash I} A e(u) \cdot e(\varphi) d x \\
= & -\left.\int_{I} A e(u) e_{2}\right|_{-} ^{+} \cdot \varphi d x_{1}-\int_{\Omega \backslash I}(\operatorname{div} A e(u)) \cdot \varphi d x+\int_{\partial \Omega \backslash \Gamma} A e(u) n \cdot \varphi d \sigma
\end{aligned}
$$

где $n$ - внешняя единичная нормаль на $\partial \Omega,\left.v\right|_{-} ^{+}-$разность предельных значений $\left.v\right|_{I}$ со стороны верхней и нижней полуплоскостей.

Из интегрального тождества (2.14) после интегрирования по частям на отрезке $I$ получаем равенство

$$
\int_{\Omega} A e(u) \cdot e(\varphi) d x=b \int_{I} \frac{\partial^{2} u_{1}}{\partial x_{1}^{2}} \varphi_{1} d x_{1}-\left.b \frac{\partial u_{1}}{\partial x_{1}} \varphi_{1}\right|_{M}+\int_{\Omega} f \cdot \varphi d x+\int_{I} g \cdot \varphi d x_{1}
$$

где $M$ - точка с координатами $(1,0)$.

Сравнивая правые части последних двух равенств, в силу произвольности $\varphi$ выводим соотношения, которьм решение $u$ удовлетворяет в области $\Omega$, на отрезке $I$ и в их граничных точках:

$$
\begin{gathered}
-\operatorname{div} A e(u)=f \text { в } \Omega \backslash I, \\
-b \frac{\partial^{2} u_{1}}{\partial x_{1}^{2}} e_{1}=\left.A e(u) e_{2}\right|_{-} ^{+}+g \text { на } I, \quad A e(u) n=0 \text { на } \partial \Omega \backslash(\Gamma \cup M), \\
u=0 \text { на } \Gamma,\left.\frac{\partial u_{1}}{\partial x_{1}}\right|_{M}=0 .
\end{gathered}
$$

Получили смешанную задачу в области $\Omega \backslash I$ с краевьм условием типа Вентцеля на внутренней границе $I$ и краевым условием типа Дирихле и Неймана на внешней гранище $\partial \Omega$. 
Пример более сложной составной структуры. Рассмотрим плиту $\Omega=$ $(0,1) \times(-1 / 2,1 / 2)$ с двумя стержнями $I_{1}, I_{2}$, где $I_{1}=(0,1) \times\{0\}, I_{2}=\{1 / 2\} \times$ $(-1 / 2,1 / 2)$, и пусть мера $\lambda$, сосредоточенная на "кресте" $I_{1} \cup I_{2}$, задана соотношением: $d \lambda=d x_{1}$ на $I_{1}, d \lambda=d x_{2}$ на $I_{2}$.

Для задачи (2.2) с такой мерой $\lambda$ классическая форма уравнения Эйлера имеет вид следуюшей смешанной задачи:

$$
\begin{gathered}
-\operatorname{div} A e(u)=f \text { в } \Omega \backslash\left(I_{1} \cup I_{2}\right), \\
-b_{1} \frac{\partial^{2} u_{1}}{\partial x_{1}^{2}} e_{1}=\left.A e(u) e_{2}\right|_{-} ^{+}+g \text { на } I_{1} \backslash O, \\
-b_{2} \frac{\partial^{2} u_{2}}{\partial x_{2}^{2}} e_{2}=\left.A e(u) e_{1}\right|_{-} ^{+}+g \text { на } I_{2} \backslash O, \\
\left.\frac{\partial u_{1}}{\partial x_{1}}\right|_{I_{1}},\left.\quad \frac{\partial u_{2}}{\partial x_{2}}\right|_{I_{2}} \text { непрерывны в точке } O, \\
A e(u) n=0 \text { на } \partial \Omega \backslash\left(\Gamma \cup M_{1} \cup M_{2} \cup M_{3}\right), \quad u=0 \text { на } \Gamma, \\
\left.\frac{\partial u_{1}}{\partial x_{1}}\right|_{M_{1}}=0,\left.\quad \frac{\partial u_{2}}{\partial x_{2}}\right|_{M_{2} \cup M_{3}}=0 .
\end{gathered}
$$

Здесь $b_{i}=\left(B^{-1} \eta_{i} \cdot \eta_{i}\right)^{-1}, \eta_{i}=e_{i} \times e_{i}, i=1,2 ; O=I_{1} \cap I_{2}$, а точки $M_{1}, M_{2}, M_{3}$ имеют координаты $(0,1),(1 / 2,1 / 2),(1 / 2,-1 / 2)$ соответственно.

\section{§3. Задача на плите с тонким стержнем}

На квадрате $\Omega=(0,1) \times(-1 / 2,1 / 2)$ с выделенной полосой $I^{h}=(0,1) \times$ $(-h / 2, h / 2)$ введем меру

$$
d \mu^{h}=\left.d x\right|_{\Omega}+d \lambda^{h}, \quad \text { где } d \lambda^{h}=\left.\frac{1}{h} d x\right|_{I^{h}} .
$$

Имеется слабая сходимость мер $\lambda^{h} \rightarrow \lambda, \mu^{h} \rightarrow \mu(\lambda, \mu$ определены в (2.1)), что означает, например: $\int_{\Omega} \varphi d \lambda^{h} \rightarrow \int_{\Omega} \varphi d \lambda \forall \varphi \in C_{0}(\Omega)$.

Определим пространство $V=\left\{u \in H^{1}(\Omega)^{2}:\left.u\right|_{x_{1}=0}=0\right\}$, в котором плотно множество функций $C_{\Gamma}^{\infty}(\bar{\Omega})^{2}$ из $\S 2$, и рассмотрим задачу на отыскание энергии

$$
J^{h}=\min _{u \in V} \int_{\Omega}\left(\mathscr{A}^{h} e(u) \cdot e(u)-2 G^{h} \cdot u\right) d \mu^{h} .
$$

Здесь тензор упругости $\mathscr{A}^{h}$ и вектор-функция $G^{h}$ представлены парой компонент

$$
\mathscr{A}^{h}=(A, B), \quad G^{h}=\left(f^{h}, g^{h}\right),
$$

первая из них “действует” в квадрате $\Omega$, вторая - на полосе $I^{h}, f^{h} \in L^{2}(\Omega, d x)^{2}$, $g^{h} \in L^{2}\left(\Omega, d \lambda^{h}\right)^{2}$. По определению

$$
\int_{\Omega} G^{h} \cdot u d \mu^{h}=\int_{\Omega} f^{h} \cdot u d x+\int_{\Omega} g^{h} \cdot u d \lambda^{h} .
$$


Предполагаем, что тензоры $A, B$ - те же, что в $\S 2$, вектор $g^{h}$ имеет продольное направление, т.е.

$$
g^{h}=\left(g_{1}^{h}, 0\right)
$$

и пусть

$$
G^{h} \rightarrow G \text { в } L^{2}\left(\Omega, d \mu^{h}\right)^{2} .
$$

Сходимость (3.3) эквивалентна тому, что

$$
f^{h} \rightarrow f \text { в } L^{2}(\Omega, d x)^{2}, \quad g^{h} \rightarrow g \text { в } L^{2}\left(\Omega^{h}, d \lambda^{h}\right)^{2} .
$$

Здесь возникает слабая сходимость в “переменном" пространстве $L^{2}\left(\Omega, d \lambda^{h}\right)$. Напомним [19], [20], что последовательность $v^{h} \in L^{2}\left(\Omega, d \lambda^{h}\right)$ называется ограниченной, если

$$
\limsup _{h \rightarrow 0} \int_{\Omega}\left|v^{h}\right|^{2} d \lambda^{h}<\infty,
$$

и слабо сходящейся, если она ограничена и найдется элемент $v \in L^{2}(\Omega, d \lambda)$ такой, что

$$
\lim _{h \rightarrow 0} \int_{\Omega} v^{h} \varphi d \lambda^{h}=\int_{\Omega} v \varphi d \lambda \quad \forall \varphi \in C_{0}^{\infty}(\Omega) .
$$

Выполнено следующее свойство: из ограниченной в $L^{2}\left(\Omega, d \lambda^{h}\right)$ последовательности можно выделить подпоследовательность, слабо сходящуюся в $L^{2}\left(\Omega, d \lambda^{h}\right)$.

Сильная сходимость $v^{h} \rightarrow v \in L^{2}(\Omega, d \lambda)$ в $L^{2}\left(\Omega, d \lambda^{h}\right)$ означает, что

$$
\lim _{h \rightarrow 0} \int_{\Omega} v^{h} g^{h} d \lambda^{h}=\int_{\Omega} v g d \lambda,
$$

как только $g^{h} \rightarrow g$ в $L^{2}\left(\Omega, d \lambda^{h}\right)$.

Вернемся к задаче (3.1). Ее минимизант удовлетворяет интегральному тождеству

$$
u^{h} \in V, \quad \int_{\Omega} \mathscr{A}^{h} e\left(u^{h}\right) \cdot e(\varphi) d \mu^{h}=\int_{\Omega} G^{h} \cdot \varphi d \mu^{h} \quad \forall \varphi \in V,
$$

что в более детальной записи означает:

$$
\int_{\Omega} \mathscr{A} e\left(u^{h}\right) \cdot e(\varphi) d x+\int_{\Omega} B e\left(u^{h}\right) \cdot e(\varphi) d \lambda^{h}=\int_{\Omega} f^{h} \cdot \varphi d x+\int_{\Omega} g^{h} \cdot \varphi d \lambda^{h} \quad \forall \varphi \in V .
$$

Поскольку в задаче (3.4) мера $d \mu^{h}$ абсолютно непрерывна относительно меры Лебега, проблем с сушествованием и единственностью решения нет.

Изучим задачу (3.1) при $h \rightarrow 0$. Покажем, что предельной для нее является задача (2.5).

Сначала из интегрального тождества (3.4), учитывая (3.2), (2.8), а также неравенство Фридрихса на полосе $I^{h}$ :

$$
\int_{I^{h}} \varphi_{1}^{2} d x \leqslant \int_{I^{h}}\left|\frac{\partial \varphi_{1}}{\partial x_{1}}\right|^{2} d x=\int_{I^{h}}\left|e_{11}(\varphi)\right|^{2} d x, \quad \varphi \in V
$$


проверяем условие ограниченности для ее решения:

$$
\limsup _{h \rightarrow 0} \int_{\Omega}\left(\left|u^{h}\right|^{2}+\left|\nabla u^{h}\right|^{2}\right) d x<\infty, \quad \limsup _{h \rightarrow 0} \int_{\Omega}\left(\left|u_{1}^{h}\right|^{2}+\left|e\left(u^{h}\right)\right|\right)^{2} d \lambda^{h}<\infty .
$$

Благодаря (3.5)1 можно считать, что

$$
u^{h} \rightarrow u \text { в } H^{1}(\Omega)^{2},
$$

поэтому

$$
u \in V, \quad e\left(u^{h}\right) \rightarrow e(u) \text { в } L^{2}(\Omega)^{3} .
$$

Тогда после перехода к переделу в (3.4) получим

$$
v \in V, \quad \int_{\Omega} A e(u) \cdot e(\varphi) d x+\lim _{h \rightarrow 0} \int_{I^{h}} B e\left(u^{h}\right) \cdot e(\varphi) d \lambda^{h}=\int_{\Omega} G \cdot \varphi d \mu \quad \forall \varphi \in V .
$$

Изучим второе слагаемое в (3.8). В силу $(3.5)_{2}$

$$
\left(u_{1}^{h}, 0\right), \quad e\left(u^{h}\right) \text { ограничены в } L^{2}\left(\Omega, \lambda^{h}\right),
$$

поэтому (переходя к подпоследовательности) можно считать, что

$$
\begin{gathered}
\left(u_{1}^{h}, 0\right) \rightarrow \widetilde{u} \in L^{2}(\Omega, d \lambda)^{2} \quad \text { в } L^{2}\left(\Omega, d \lambda^{h}\right)^{2}, \\
e\left(u^{h}\right) \rightarrow z \in L^{2}(\Omega, d \lambda)^{3} \quad \text { в } L\left(\Omega, d \lambda^{h}\right)^{3} .
\end{gathered}
$$

Полезны следующие две леммы, доказательство которых пока отложим.

ЛЕмма 3.1. Пусть последовательность вектор-функиий $u^{h}$ удовлетворяет соотношениям (3.9), (3.10). Тогда

(i) $\widetilde{u}_{1} \in H^{1}(I)$;

(ii) $z=е(\widetilde{u})$ в смысле пространства $\mathscr{H}(\Omega, d \lambda)$, т.е.

$$
z=\left(\begin{array}{cc}
\frac{\partial \widetilde{u}_{1}}{\partial x_{1}} & \alpha \\
\alpha & \beta
\end{array}\right), \quad \alpha, \beta \in L^{2}(\Omega, d \lambda)
$$

ЛЕмма 3.2. Ecли $v^{h} \rightarrow v$ в $H^{1}(\Omega)$, mo $\left.v^{h} \rightarrow v\right|_{I}$ в $L^{2}\left(\Omega, d \lambda^{h}\right)$, әде $\left.v\right|_{I}-c л е \partial$ функиии $v \in H^{1}(\Omega)$.

Согласно лемме 3.2 можно отождествить предельную функцию $\widetilde{u}_{1}$ из (3.10) и след $\left.u_{1}\right|_{I}$ функции $u_{1} \in H^{1}(\Omega)$ из (3.6). Поэтому в силу (3.10), (3.11) второе слагаемое из (3.8) равно $\int_{I} B e(\widetilde{u}) \cdot e(\varphi) d \lambda$, где

$$
\begin{gathered}
e(\widetilde{u})=\left(\begin{array}{cc}
\frac{\partial \widetilde{u}_{1}}{\partial x_{1}} & \alpha \\
\alpha & \beta
\end{array}\right)=\left(\begin{array}{cc}
\frac{\partial u_{1}}{\partial x_{1}} & \alpha \\
\alpha & \beta
\end{array}\right)=e^{t}(u)+g, \\
e^{t}(u)=\left(\begin{array}{cc}
\frac{\partial u_{1}}{\partial x_{1}} & 0 \\
0 & 0
\end{array}\right), \quad g=\left(\begin{array}{cc}
0 & \alpha \\
\alpha & \beta
\end{array}\right) \in \mathscr{E}(0),
\end{gathered}
$$


и тождество (3.8) имеет вид

$$
\int_{\Omega} A e(u) \cdot e(\varphi) d x+\int_{I} B\left(e^{t}(u)+g\right) \cdot e(\varphi) d \lambda=\int_{\Omega} G \cdot \varphi d \mu \quad \forall \varphi \in V .
$$

Подставив в тождество (3.13) функции $\varphi=\varphi^{n}$, реализующие произвольный градиент нуля $p \in \mathscr{E}(0)$, такие, что $\varphi^{n} \rightarrow 0, e\left(\varphi^{n}\right) \rightarrow p$ в $L^{2}\left(\Omega, d \lambda^{h}\right)$, в пределе получим равенство

$$
\int_{I} B\left(e^{t}(u)+g\right) \cdot p d \lambda=0 \quad \forall p \in \mathscr{E}(0),
$$

которое влечет тангенциальность матрицы $B\left(e^{t}(u)+g\right)$, т.е. $B\left(e^{t}(u)+g\right) \in \mathscr{E}(0)^{\perp}$.

Отсюда по лемме 2.1 получаем равенство

$$
B\left(e^{t}(u)+g\right) \cdot p=0 \quad \forall p \in \mathscr{E}(0) \quad \lambda \text {-п.в. },
$$

которое есть уравнение Эйлера для минимизанта $g(\xi)$ (где $\xi=e^{t}(u)$ ) вариационной задачи, определяюшей тензор релаксации $\widehat{B}$. Упростим второе слагаемое в (3.13), используя свойства тензора релаксации $\widehat{B}$. Из $(3.14)$ и свойств $(2.13)_{3}$, $(2.13)_{1}$ для тензора релаксации $\widehat{B}$ выводим представление

$$
B\left(e^{t}(u)+g\right)=\widehat{B}\left(e^{t}(u)+g\right)=\widehat{B} e^{t}(u) .
$$

Отсюда, учитывая $(2.12),(2.13)_{2}$ и структуру тангенциального градиента $e^{t}(u)$, получаем равенства

$$
\begin{aligned}
\int_{I} B\left(e^{t}(u)+g\right) \cdot e(\varphi) d \lambda & =\int_{I} \widehat{B} e^{t}(u) \cdot e(\varphi) d \lambda=\int_{I} \widehat{B} e^{t}(u) \cdot e^{t}(\varphi) d \lambda \\
& =\int_{I} b(y) \frac{\partial u_{1}}{\partial x_{1}} \frac{\partial \varphi_{1}}{\partial x_{1}} d \lambda .
\end{aligned}
$$

Тогда тождество (3.13) принимает вид

$$
\int_{\Omega} A e(u) \cdot e(\varphi) d x+\int_{I} b \frac{\partial u_{1}}{\partial x_{1}} \frac{\partial \varphi_{1}}{\partial x_{1}} d \lambda=\int_{\Omega} G \cdot \varphi d \mu \quad \forall \varphi \in C_{\Gamma}^{\infty}(\bar{\Omega}) .
$$

Но это в точности тождество (2.14) для минимизанта задачи (2.5).

Из сделанных замечаний вытекает

ТЕОРема 3.3. Пусть выполнены предположения (3.2), (3.3), u, $u^{h}$-решения задач (2.6), (3.4). Тогда

$$
u^{h} \rightarrow u \quad \text { в } H^{1}(\Omega)^{2},\left.\quad u_{1}^{h} \rightarrow u_{1}\right|_{I} \quad \text { в } \quad L^{2}\left(\Omega, d \lambda^{h}\right),
$$

где $\left.u_{1}\right|_{I}-$ след функиии $u_{1} \in H^{1}(\Omega)$.

Можно показать, что если слабую сходимость (3.3) заменить на сильную сходимость в $L^{2}\left(Y, d \mu^{h}\right)^{2}$, то будут сходиться энергии $J^{h} \rightarrow J$ (см. $\left.(3.1),(3.2)\right)$, а в (3.15) также можно перейти к соответствующим сильным сходимостям. Подробные выводы аналогичных утверждений приводятся далее для более сложных задач (см. $\S 7,8)$.

Наконец, докажем леммы 3.1 и 3.2. Справедливо следующее 
ПРЕДЛОЖЕНИЕ 3.4. Если $g^{h} \rightarrow g$ в $L^{2}\left(\Omega, d \lambda^{h}\right)$, mo

$$
\bar{g}^{h} \equiv \frac{1}{h} \int_{-h / 2}^{h / 2} g\left(x_{1}, x_{2}\right) d x_{2} \rightarrow g \quad \text { в } \quad L^{2}\left(I, d x_{1}\right)
$$

Несложное доказательство его опускаем.

Согласно (3.10)

$$
u_{1}^{h} \rightarrow u_{1}, \quad \frac{\partial u_{1}^{h}}{\partial x_{1}} \rightarrow z_{11} \text { в } L^{2}\left(\Omega, d \lambda^{h}\right)
$$

и, значит, по предложению 3.4 сходятся поперечные средние

$$
\bar{u}_{1}^{h} \rightarrow \bar{u}_{1}, \quad \frac{\partial}{\partial x_{1}} \bar{u}_{1}^{h}=\frac{1}{h} \int_{-h / 2}^{h / 2} \frac{\partial u_{1}^{h}}{\partial x_{1}} d x_{2} \rightarrow z_{11} \text { в } L^{2}\left(I, d x_{1}\right),
$$

откуда следуют все утверждения леммы 3.1 .

Лемма 3.2 выводится из классической теоремы о следе функции $v \in H^{1}(\Omega)$

$$
\frac{1}{h} \int_{I^{h}} v^{2} d \lambda^{h} \leqslant \delta \int_{\Omega}|\nabla v|^{2} d x+C_{\delta} \int_{\Omega}|v|^{2} d x
$$

где константа $C_{\delta}$ не зависит от $h$.

\section{§4. Задача на плите с сингулярной сеткой}

Рассмотрим плиту с армированием в виде $\varepsilon$-периодической сингулярной сетки. Для простоты пусть это будет квадратная сетка $F_{\varepsilon}$, введенная в $\S 1$. Обозначим через $F_{1, \varepsilon}\left(F_{2, \varepsilon}\right)$ множество всех горизонтальных (вертикальных) прямых из $F_{\varepsilon}$.

Соболевское пространство. По схеме, изложенной в $\S 2$, определим для меры $\mu_{\varepsilon}$ и области $\Omega$ пространства $W_{\varepsilon}, \mathscr{H}\left(\Omega, d \mu_{\varepsilon}\right)$, а также множества градиентов $\mathscr{E}(u), \mathscr{E}(0)$ и тангенциальных матриц $\mathscr{E}(0)^{\perp}$. Опуская подробности, выделим некоторые моменты. Например, $W_{\varepsilon}$ - замыкание множества $\left\{(u, e(u)): u \in C_{0}^{\infty}(\Omega)^{2}\right\}$ в $L^{2}\left(\Omega, d \mu_{\varepsilon}\right)^{2} \times L^{2}\left(\Omega, d \mu_{\varepsilon}\right)^{3}$. Элементами пространства $W_{\varepsilon}$ служат пары $(u, v), u-$ вектор, $v$ - матрица. Матрицу $v$ называем симметрическим градиентом (или просто градиентом) вектора $u$ и обозначаем через $e(u)$.

По определению $z \in \mathscr{E}(0)$, если сушествует последовательность $\varphi^{n} \in C_{0}^{\infty}(\Omega)^{2}$ такая, что

$$
\varphi^{n} \rightarrow 0 \quad \text { в } L^{2}\left(\Omega, d \mu_{\varepsilon}\right)^{2}, \quad e\left(\varphi^{n}\right) \rightarrow z \quad \text { в } L^{2}\left(\Omega, d \mu_{\varepsilon}\right)^{3} .
$$

Нетривиальность градиента нуля $z \not \equiv 0$ проявляется только на сетке $F_{\varepsilon}$, причем $\left.z\right|_{F_{1, \varepsilon}}=\left(\begin{array}{cc}0 & \alpha \\ \alpha & \beta\end{array}\right),\left.z\right|_{F_{2, \varepsilon}}=\left(\begin{array}{cc}\beta & \alpha \\ \alpha & 0\end{array}\right)$, где $\alpha, \beta$ - произвольные функции из $L^{2}\left(F_{1, \varepsilon}\right)$ или $L^{2}\left(F_{2, \varepsilon}\right)$.

Любой градиент $e(u)$ допускает единственное представление в виде суммы градиента нуля и тангенциальной матрицы

$$
e(u)=e^{t}(u)+z, \quad z \in \mathscr{E}(0), \quad e^{t}(u) \perp \mathscr{E}(0),
$$


и компоненту $e^{t}(u)$ называют тангенциальным градиентом функции $u$. Структура множества $\mathscr{E}(0)$ определяет структуру тангенциального градиента

$$
e^{t}(u)=\left\{\begin{array}{lc}
e(u) & \text { вне } F_{\varepsilon}, \\
\left(\begin{array}{cc}
\frac{\partial u_{1}}{\partial x_{1}} & 0 \\
0 & 0
\end{array}\right) & \text { на } F_{1, \varepsilon}, \\
\left(\begin{array}{cc}
0 & 0 \\
0 & \frac{\partial u_{2}}{\partial x_{2}}
\end{array}\right) & \text { на } F_{2, \varepsilon} .
\end{array}\right.
$$

Можно дать описание пространства $\mathscr{H}\left(\Omega, d \mu_{\varepsilon}\right)=\left\{u:(u, v) \in W_{\varepsilon}\right\}$ в терминах обычных соболевских пространств с лебеговой мерой. Принадлежность функции $u=\left(u_{1}, u_{2}\right)$ пространству $\mathscr{H}\left(\Omega, d \mu_{\varepsilon}\right)$ означает, что $u \in H_{0}^{1}(\Omega)^{2}$ и если $\left.u_{1}\right|_{F_{\varepsilon}},\left.u_{2}\right|_{F_{\varepsilon}}$ - следы функций $u_{1}, u_{2} \in H_{0}^{1}(\Omega)$ на сетку $F_{\varepsilon}$, то $\left.u_{i}\right|_{F_{i, \varepsilon}} \in H^{1}\left(F_{i, \varepsilon}\right), i=1,2$.

Вернемся к задаче (1.4). Ясно, что $J^{\varepsilon}=\min _{(u, v) \in W_{\varepsilon}} \int_{\Omega}(A v \cdot v-2 f \cdot u) d \mu_{\varepsilon}$ и минимизант этой задачи - пара, состоящая из функции $u^{\varepsilon}$ и ее градиента $e\left(u^{\varepsilon}\right)$.

Для минимизанта $\left(u^{\varepsilon}, e\left(u^{\varepsilon}\right)\right)$ имеем уравнение Эйлера в виде вариационного тождества

$$
\int_{\Omega} A e\left(u^{\varepsilon}\right) \cdot e(\varphi) d \mu_{\varepsilon}=\int_{\Omega} f \cdot \varphi d \mu_{\varepsilon} \quad \forall \varphi \in C_{0}^{\infty}(\Omega)^{2} .
$$

Вследствие неравенства Корна (1.5) с $h=0$ в тождестве (4.3) левая часть задает скалярное произведение в пространстве $W_{\varepsilon}$, а правая часть - непрерьвньй функционал по соответствуюшей норме, называемой энергетической. В энергетической норме участвует только вторая компонента пары $(u, e(u)) \in W_{\varepsilon}$. Но по второй компоненте пара определяется однозначно (см. (1.5)). Отсюда по теореме Рисса выводим существование и единственность минимизанта задачи (1.4), а также условие ограниченности

$$
\limsup _{\varepsilon \rightarrow 0} \int_{\Omega}\left(\left|u^{\varepsilon}\right|^{2}+\left|e\left(u^{\varepsilon}\right)\right|^{2}\right) d \mu_{\varepsilon}<\infty .
$$

Тензор релаксации. Пусть $A$ - положительно определенный симметрический тензор (оператор), действуюший в пространстве симметрических матриц $\xi=$ $\left\{\xi_{i j}\right\}_{i, j=1}^{2}$. Аналогично, как в $\S 2$, введем для него тензор релаксации на структуpe $F_{\varepsilon}$.

По определению на звеньях с направляющим ортом $e_{i}, i=1,2$, тензор релаксации $\widehat{A}$ совпадает с тензором $\widehat{A}_{i}$, который определяется соотношением

$$
\widehat{A}_{i} \xi \cdot \xi=\min _{\xi \in \mathscr{E}_{i}} A(\xi+\xi) \cdot(\xi+\xi), \quad \text { где } \mathscr{E}_{i}=\left\{\xi: \xi \perp e_{i} \times e_{i}\right\} .
$$

Несложные вычисления показывают, что

$\widehat{A}_{1} \xi \cdot \xi=a_{1} \xi_{11}^{2}, \quad \widehat{A}_{2} \xi \cdot \xi=a_{2} \xi_{22}^{2}, \quad$ где $a_{i}=\left(A^{-1} \eta_{i} \cdot \eta_{i}\right)^{-1}, \quad \eta_{i}=e_{i} \times e_{i}, \quad i=1,2$.

В случае изотропного тензора (см. (1.2)) $a_{1}=a_{2}=\widehat{k}=k\left(k+2 k_{1}\right) /\left(k+k_{1}\right)$.

Перечислим некоторые свойства тензора $\widehat{A}_{i}$, вытекающие из его определения: $\operatorname{Ker} \widehat{A}_{i}=\mathscr{E}_{i} ; \mathscr{E}_{i}^{\perp}-$ область значений $\widehat{A}_{i} ; A \xi=\widehat{A}_{i} \xi$, если $A \xi \in \mathscr{E}_{i}^{\perp}$. 
Классическая форма уравнения Эйлера. Тождество (4.3) в более подробной записи означает, что

$$
\int_{\Omega} A e\left(u^{\varepsilon}\right) \cdot e(\varphi) d x+\int_{F_{\varepsilon}} A e\left(u^{\varepsilon}\right) \cdot e(\varphi) d \lambda_{\varepsilon}=2 \int_{\Omega} f \cdot \varphi d \mu_{\varepsilon} .
$$

Подставив в него $\varphi=\varphi^{n}$ из (4.1), после перехода к пределу (по $n$ ) получим условие

$$
\operatorname{Ae}\left(u_{\varepsilon}\right) \perp z \quad \forall z \in \mathscr{E}(0) .
$$

Отсюда, используя разложение (4.2) и свойства тензора релаксации $\widehat{A}$, можно переписать тождество в терминах тангенциального градиента:

$$
\int_{\Omega} A e\left(u^{\varepsilon}\right) \cdot e(\varphi) d x+\int_{F_{\varepsilon}} \widehat{A} e^{t}\left(u^{\varepsilon}\right) \cdot e^{t}(\varphi) d \lambda_{\varepsilon}=2 \int_{\Omega} f \cdot \varphi d \mu_{\varepsilon}
$$

что с учетом (4.5) и определения меры $\lambda_{\varepsilon}(\mathrm{cm.} \S 1)$ дает тождество

$$
\begin{aligned}
\int_{\Omega} & A e\left(u^{\varepsilon}\right) \cdot e(\varphi) d x+\frac{\varepsilon}{2} \int_{F_{1, \varepsilon}} a_{1} \frac{\partial u_{1}^{\varepsilon}}{\partial x_{1}} \frac{\partial \varphi_{1}}{\partial x_{1}} d x_{1}+\frac{\varepsilon}{2} \int_{F_{2, \varepsilon}} a_{2} \frac{\partial u_{2}^{\varepsilon}}{\partial x_{2}} \frac{\partial \varphi_{2}}{\partial x_{2}} d x_{2} \\
= & 2 \int_{\Omega} f \cdot \varphi d \mu_{\varepsilon} .
\end{aligned}
$$

После интегрирования по частям можно получить классическую постановку задачи для $u^{\varepsilon}$ :

$$
\begin{gathered}
-\operatorname{div} A e\left(u^{\varepsilon}\right)=f \text { в } \Omega \backslash F_{\varepsilon}, \\
-a_{1} \frac{\partial^{2} u_{1}^{\varepsilon}}{\partial x_{1}^{2}} e_{1}=\left.\frac{2}{\varepsilon} A e\left(u^{\varepsilon}\right) e_{2}\right|_{-} ^{+}+f \text { на } F_{1, \varepsilon} \cap \Omega \text { вне узлов, } \\
-a_{2} \frac{\partial^{2} u_{2}^{\varepsilon}}{\partial x_{2}^{2}} e_{2}=\left.\frac{2}{\varepsilon} A e\left(u^{\varepsilon}\right) e_{1}\right|_{-} ^{+}+f \text { на } F_{2, \varepsilon} \cap \Omega \text { вне узлов, } \\
\left.\frac{\partial u_{1}^{\varepsilon}}{\partial x_{1}}\right|_{F_{1, \varepsilon}} \text { и }\left.\frac{\partial u_{2}^{\varepsilon}}{\partial x_{2}}\right|_{F_{2, \varepsilon}} \text { непрерывны в узлах, }\left.\quad u^{\varepsilon}\right|_{\partial \Omega}=0 .
\end{gathered}
$$

Здесь выражение $\left.v\right|_{-} ^{+}$на прямой из $F_{1, \varepsilon}$ означает разность предельных значений $v$ на прямой со стороны верхней и нижней полуплоскостей. Аналогичный смысл придается выражению $\left.v\right|_{-} ^{+}$на прямой из $F_{2, \varepsilon}$.

\section{§5. Пространства периодических функций}

В этом параграфе изучена введенная в $\S 1$ периодическая составная структура с квадратной сеткой.

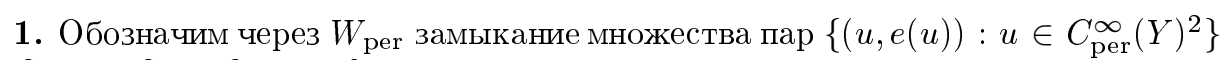
в $L^{2}(Y, d \mu)^{2} \times L^{2}(Y, d \mu)^{3}$. Далее, аналогично, как в $\S \S 2,4$, можно определить и 
описать пространства $\mathscr{H}_{\text {per }}(Y, d \mu)$, а также множества градиентов $\mathscr{E}(0)$ и тангенциальных матриц $\mathscr{E}(0)^{\perp}$. Например, если пара вектор-функций $u,\left.u\right|_{F}$, заданных на $Y$ и $F$ соответственно, представляет элемент из $\mathscr{H}_{\mathrm{per}}(Y, d \mu)$, то

$$
\begin{gathered}
u=\left(u_{1}, u_{2}\right) \in H_{\mathrm{per}}^{1}(Y, d x)^{2}, \\
\left.u_{1}\right|_{F},\left.u_{2}\right|_{F}-\text { следы функций } u_{1}, u_{2} \in H_{\mathrm{per}}^{1}(Y, d x), \\
\left.u_{1}\right|_{I_{1}} \in H^{1}\left(I_{1}\right),\left.\quad u_{2}\right|_{I_{2}} \in H^{1}\left(I_{2}\right) \text { на отрезках } I_{1}, I_{2} .
\end{gathered}
$$

Для теории усреднения важно выяснить структуру пространства $\mathscr{R}$ периодических жестких перемешений. Введем это ключевое понятие.

ОПРЕДЕЛЕНИЕ 5.1. Вектор $u \in \mathscr{H}_{\text {per }}(Y, d \mu)$ называется периодическим жестким перемещением, если $0 \in \mathscr{E}(u)$ (т.е. среди градиентов вектора $u$ есть нулевой).

Очевидно, что пространство $\mathscr{R}$ для любой меры содержит постоянные векторы и в случае меры Лебега оно исчерпывается этими векторами. Если же вместо $\mu$ рассматривать меру $\lambda$, сосредоточенную на сетке $F$, то пространство $\mathscr{R}$ оказывается шире множества постоянных векторов. Любой элемент $u \in \mathscr{R}$ допускает единственное представление $[3 ; \S 6]$

$$
u=C+g
$$

где $C$ - постоянный вектор, а $g$ - поперечное перемешение, т.е. вектор, который на каждом звене сетки $F$ ортогонален этому звену. Отсюда для составной меры $\mu$ из (1.1) вектор $u \in \mathscr{R}$ удовлетворяет условиям:

$$
\begin{gathered}
u-\text { постоянный вектор вне } F, \\
\left.u\right|_{F} \quad \text { допускает представление }(5.2), \\
\left.u\right|_{F}-\text { след функции } u \in H^{1}(Y, d x)^{2} \text { в силу }(5.1)_{2} \cdot
\end{gathered}
$$

Перечисленные три условия можно совместить, если $u=C$ всюду на ячейке периодичности $Y$. Итак, доказано

ПРЕДЛОЖЕНИЕ 5.2. В пространстве $\mathscr{H}_{\text {per }}(Y, d \mu)$ множсество $\mathscr{R}$ исчерпьвается постоянными векторами.

Это - ключевой факт, определяющий классический характер усреднения задачи $(1.4)$, а также задачи (1.3) с тонкой сеткой $F_{\varepsilon}^{h}$, когда $h=h(\varepsilon) \rightarrow 0$.

2. Введем сходимость в “переменном" пространстве периодических функций $L^{2}\left(Y, d \mu^{h}\right)$ (см. [3; $\left.\left.\S 11\right],[20]\right)$. Пусть $u^{h} \in L^{2}\left(Y, d \mu^{h}\right)$ и

$$
\limsup _{h \rightarrow 0} \int_{Y}\left|u^{h}\right|^{2} d \mu^{h}<\infty .
$$

Скажем, что $u^{h} \rightarrow u \in L^{2}(Y, d \mu)$ в $L^{2}\left(Y, d \mu^{h}\right)$, если

$$
\int_{Y} u^{h} \varphi d \mu^{h} \rightarrow \int_{Y} u \varphi d \mu \quad \forall \varphi \in C_{\mathrm{per}}^{\infty}(Y) .
$$


Сходимость $u^{h} \rightarrow u \in L^{2}(Y, d \mu)$ в $L^{2}\left(Y, d \mu^{h}\right)$ означает, что

$$
\int_{Y} u^{h} v^{h} d \mu^{h} \rightarrow \int_{Y} u v d \mu, \quad \text { как только } v^{h} \rightarrow v \text { в } L^{2}\left(Y, d \mu^{h}\right) .
$$

Напомним, что вектор $a \in L^{2}(Y, d \mu)^{2}$ и симметричная матрица $b \in L^{2}(Y, d \mu)^{3}$ удовлетворяют соотношению

$$
a=\operatorname{div} b \quad(\text { в смысле меры } \mu),
$$

если $\int_{Y} b \cdot e(\varphi) d \mu=-\int_{Y} a \cdot \varphi d \mu$ для любого $\varphi \in C_{\mathrm{per}}^{\infty}(Y)^{2}$. В случае $a \equiv 0$ это определение соленоидальной матрицы $b, b \in V_{\mathrm{sol}}(Y, d \mu)$.

Аналогично, вектор $a^{h} \in L^{2}\left(Y, d \mu^{h}\right)^{2}$ и матрица $b^{h} \in L^{2}\left(Y, d \mu^{h}\right)^{3}$ удовлетворяют соотношению

$$
\left.a^{h}=\operatorname{div} b^{h} \quad \text { (в смысле меры } \mu^{h}\right),
$$

если

$$
\int_{Y} b^{h} \cdot e(\varphi) d \mu^{h}=-\int_{Y} a^{h} \cdot \varphi d \mu^{h} \quad \forall \varphi \in C_{\mathrm{per}}^{\infty}(Y)^{2} .
$$

В случае $a^{h} \equiv 0$ получаем матрицу $b^{h}$, соленоидальную относительно меры $\mu^{h}$.

ОПРЕДЕЛЕНИЕ 5.3. Скажем, что меры $\mu^{h}$ и $\mu$ связаны аппроксимативными условиями, если

(i) для любых $a$ и $b$, удовлетворяюших соотношению (5.4), найдутся $a^{h}$ и $b^{h}$, удовлетворяюшие соотношению (5.5), при этом

$$
a^{h} \rightarrow a \text { в } L^{2}\left(Y, d \mu^{h}\right)^{2}, \quad b^{h} \rightarrow b \quad \text { в } L^{2}\left(Y, d \mu^{h}\right)^{3} ;
$$

(ii) если в (i) $a \equiv 0$, то и $a^{h} \equiv 0$ (сильная аппроксимируемость соленоидальных матриш).

Теорема 5.4. Меры $\mu^{h}, \mu$, определенные в (1.1), связаны аппроксимативныци условиями.

ДокАЗАТЕЛЬСТво. $1^{\circ}$. Строим меру сглаживания $\widetilde{\mu}^{h}$, определенную соотношением

$$
\int_{Y} \varphi d \widetilde{\mu}^{h}=\int_{Y}(\varphi)_{h} d \mu \quad \forall \varphi \in C_{\mathrm{per}}^{\infty}(Y),
$$

где $(\varphi)_{h}$ - сглаживание с ядром $\omega(y)=\chi_{Y}(y)$ (характеристическая функция единичного квадрата $Y)$ :

$$
(\varphi)_{h}(x)=h^{-2} \int \varphi(x-y) \omega\left(h^{-1} y\right) d y
$$

Сравним меры $\mu^{h}$ и $\widetilde{\mu}^{h}$. Обе они абсолютно непрерывны относительно плоской меры Лебега:

$$
\widetilde{\mu}^{h}=\widetilde{\rho}^{h}(x) d x, \quad \mu^{h}=\rho^{h}(x) d x
$$


где $\widetilde{\rho}^{h}(x)=h^{-2} \int_{x+Y_{h}} d \mu(y), Y_{h}=[-h / 2, h / 2)^{2}$ и, значит,

$$
\begin{aligned}
& \tilde{\rho}^{h}(x)= \begin{cases}\frac{1}{2} & \text { вне } F^{h}, \\
\frac{1}{2}+\frac{1}{4 h} & \text { на } F^{h} \backslash Y_{h}, \\
\frac{1}{2}+\frac{1}{2 h} & \text { на } Y_{h},\end{cases} \\
& \rho^{h}(x)= \begin{cases}\frac{1}{2} & \text { вне } F^{h}, \\
\frac{1}{2}+\frac{1}{4 h}(1+O(h)) & \text { на } F^{h} .\end{cases}
\end{aligned}
$$

Видим, что вне $Y_{h}$ плотности $\widetilde{\rho}^{h}(x), \rho^{h}(x)$ по существу совпадают.

$2^{\circ}$. Известен общий результат [3; $\left.\S 16\right]$ : пусть $m$ - произвольная периодическая борелева мера, $\widetilde{m}^{h}$ - любое ее сглаживание (с подходящим ядром сглаживания), тогда меры $m, \widetilde{m}^{h}$ связаны аппроксимативными условиями. Отсюда и изучаемые нами меры $\mu$ и $\widetilde{\mu}^{h}$ связаны аппроксимативными условиями. Тогда если вектор $a$ и матрица $b$ удовлетворяют соотношению (5.4), то найдутся вектор $\widetilde{a}^{h}$ и матрица $\widetilde{b}^{h}$, удовлетворяющие соотношению

$$
\left.\widetilde{a}^{h}=\operatorname{div} \widetilde{b}^{h} \quad \text { (в смысле меры } \widetilde{\mu}^{h}\right),
$$

т.e.

$$
\int_{Y} \widetilde{b}^{h} \cdot e(\varphi) d \widetilde{\mu}^{h}=-\int_{Y} \widetilde{a}^{h} \cdot \varphi d \widetilde{\mu}^{h} \quad \forall \varphi \in C_{\mathrm{per}}^{\infty}(Y)^{2}
$$

Положим

$$
b^{h}=\widetilde{b}^{h} \frac{\widetilde{\rho}^{h}}{\rho^{h}}, \quad a^{h}=\widetilde{a}^{h} \frac{\widetilde{\rho}^{h}}{\rho^{h}} .
$$

Тогда очевидно, что $a^{h}, b^{h}$ удовлетворяют соотношению (5.5). Кроме того, по построению, если $a \equiv 0$, то и $a^{h} \equiv 0$, так как $\widetilde{a}^{h} \equiv 0$. Остается проверить сходимости (5.7). Но они вытекают из следующих трех утверждений.

ПрЕДЛОЖЕНИЕ 5.5. Для сходимости $g^{h} \rightarrow g$ в $L^{2}\left(Y, d \mu^{h}\right)$ достаточно, чтобы соотношение (5.3) выполнялось на пробньх функииях из мнохсества

$$
\Pi=\left\{\varphi \in C_{\mathrm{per}}^{\infty}(Y): \varphi=0 \text { в окрестности узлов сетки } F\right\} .
$$

Для доказательства этого предложения достаточно заметить, что множество П плотно в $L^{2}\left(Y, d \mu^{h}\right)$ и $L^{2}(Y, d \mu)$.

Из предложения 5.5, учитывая структуру плотностей $\widetilde{\rho}^{h}, \rho^{h}$, получаем

ПРЕДЛОЖЕНИЕ 5.6. Для мер $\widetilde{\mu}^{h}, \mu^{h}$

$$
v^{h} \rightarrow v \quad \text { в } L^{2}\left(Y, d \mu^{h}\right) \quad \Longleftrightarrow \quad v^{h} \rightarrow v \quad \text { в } \quad L^{2}\left(Y, d \widetilde{\mu}^{h}\right) .
$$

Наконец, справедливо 
ПРЕДЛОЖЕНИЕ 5.7. Если $\widetilde{g}^{h} \rightarrow g$ в $L^{2}\left(Y, d \widetilde{\mu}^{h}\right)$, mo

$$
g^{h}=\widetilde{g}^{h} \frac{\widetilde{\rho}^{h}}{\rho^{h}} \rightarrow g \quad \boldsymbol{\theta} \quad L^{2}\left(Y, d \mu^{h}\right)
$$

ДокАЗАТЕЛЬСТво. Возьмем произвольную $v^{h} \rightarrow v$ в $L^{2}\left(Y, d \mu^{h}\right)$. В силу предложения 5.6 одновременно имеется сходимость $v^{h} \rightarrow v$ в $L^{2}\left(Y, d \widetilde{\mu}^{h}\right)$. Тогда

$$
\int_{Y} g^{h} v^{h} d \mu^{h}=\int_{Y} \widetilde{g}^{h} v^{h} d \widetilde{\mu}^{h} \rightarrow \int_{Y} g v d \mu
$$

по определению сильной сходимости $\widetilde{g}^{h}$ в $L^{2}\left(Y, d \widetilde{\mu}^{h}\right)$. Но последнее соотношение означает также искомую сходимость $g^{h} \rightarrow g$ в $L^{2}\left(Y, d \mu^{h}\right)$. Предложение доказано. Вместе с тем проверены оба аппроксимативных условия и теорема 5.4 доказана.

3. Для вычисления $A^{\mathrm{hom}}$ используем формулу релаксации

$$
2 A^{\mathrm{hom}} \xi \cdot \xi=A \xi \cdot \xi+\min _{u \in \mathscr{H}_{\mathrm{per}}(Y, d \mu)} \int_{Y} \widehat{A}\left(\xi^{t}+e^{t}(u)\right) \cdot\left(\xi^{t}+e^{t}(u)\right) d \lambda,
$$

где $\xi^{t}$ - тангенциальная составляющая матрицы $\xi, e^{t}(u)$ - тангенциальный градиент. Можно заметить, что минимум здесь достигается при $e^{t}(u)=0$. Тогда $A^{\text {hom }} \xi \cdot \xi=\frac{1}{2} A \xi \cdot \xi+\frac{1}{4}\left(a_{1} \xi_{11}^{2}+a_{2} \xi_{22}^{2}\right)$, где $a_{i}$ определены в (4.5). В изотропном случае получается формула (1.13).

\section{§6. Коммутативные диаграммы предельных переходов}

В задаче (1.3) можно перейти к минимуму по обычному соболевскому пространству $H_{0}^{1}(\Omega)^{2}$, так как составная мера $\mu_{\varepsilon}^{h}$ абсолютна непрерьвна относительно меры Лебега, причем с невырожденной плотностью. Для минимизантов задач $(1.3),(1.6),(1.7)$ уравнения Эйлера задаются в виде интегральных тождеств в одном и том же пространстве

$$
\begin{array}{rlrl}
u^{\varepsilon, h} & \in H_{0}^{1}(\Omega)^{2}, \quad \int_{\Omega} A e\left(u^{\varepsilon, h}\right) \cdot e(\varphi) d \mu_{\varepsilon}^{h}=\int_{\Omega} f \cdot \varphi d \mu_{\varepsilon}^{h} & \forall \varphi \in C_{0}^{\infty}(\Omega)^{2}, \\
u^{h} & \in H_{0}^{1}(\Omega)^{2}, \quad \int_{\Omega} A_{h}^{\mathrm{hom}} e\left(u^{h}\right) \cdot e(\varphi) d x=\int_{\Omega} f \cdot \varphi d x & \forall \varphi \in C_{0}^{\infty}(\Omega)^{2}, \\
u \in H_{0}^{1}(\Omega)^{2}, \quad \int_{\Omega} A^{\mathrm{hom}} e(u) \cdot e(\varphi) d x=\int_{\Omega} f \cdot \varphi d x & \forall \varphi \in C_{0}^{\infty}(\Omega)^{2} .
\end{array}
$$

Параллельно с диаграммой предельных переходов (1.10) для энергий будем рассматривать диаграмму предельных переходов

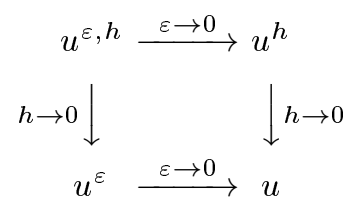


для соответствующих минимизантов, где всюду подразумевается сходимость в $L^{2}(\Omega)^{2}$.

ТЕОРема 6.1. Пусть в задачах (4.3), (6.1)-(6.3) параметрь $\varepsilon, h$ независимы. Тогда верны коммутативные диаграммы предельных переходов (6.4) и (1.10) для решений этих задач и их энергий.

В диаграммах (6.4), (1.10) рассмотрим сначала предельные переходы

$$
u^{\varepsilon, h} \rightarrow u^{h}, \quad J^{\varepsilon, h} \rightarrow J^{h} \quad \text { и } \quad u^{\varepsilon} \rightarrow u, \quad J^{\varepsilon} \rightarrow J
$$

“по горизонтали” при $\varepsilon \rightarrow 0$. Первый из них осуществляется в рамках теории усреднения относительно меры $\mu^{h}(h=$ const $>0)$, а второй - относительно меры $\mu$. В обоих случаях, хотя результаты формулируются без использования двухмасштабной сходимости, для их доказательства применяется метод двухмасштабной сходимости относительно периодической борелевой меры.

Отметим, что первый горизонтальный переход также можно осушествить, оставаясь в рамках теории усреднения в обычных лебеговых пространствах $L^{2}(\Omega)$ (с мерой Лебега $d x$ ), поскольку $d \mu^{h}=\rho^{h} d y$, где периодический множитель $\rho^{h}(y)$ принимает всего два значения: $1 / 2$ вне $F^{h}$ и $c^{h} \neq 0$ на $F^{h}$. Аналогичные задачи усреднения композитов изучались, например, в [11] методом асимптотических разложений.

Второй горизонтальньй переход оказывается обоснованным общей теоремой 8.2 , охватьвающей, в частности, и этот случай.

Разберем предельные переходы “по вертикали", т.е. при $h \rightarrow 0$. Согласно лемме 16.5 из [3] если меры $\mu^{h}, \mu$ связаны аппроксимативньми условиями, то $A_{h}^{\text {hom }} \rightarrow A^{\text {hom }}$, что позволяет обосновать $(1.9)_{2}$ и аналогичный предельный переход в диаграмме (6.4). Для этого достаточно заметить, что из интегральных тождеств (6.2), (6.3) вытекает равенство

$$
\int_{\Omega} A_{h}^{\mathrm{hom}} e\left(u^{h}-u\right) \cdot e\left(u^{h}-u\right) d x=\int_{\Omega}\left(A^{\mathrm{hom}}-A_{h}^{\mathrm{hom}}\right) e(u) \cdot e\left(u^{h}-u\right) d x .
$$

Чтобы обосновать $(1.9)_{1}$, перейдем к пределу в тождестве (6.1) при $h \rightarrow 0$. Предварительно отметим условие ограниченности

$$
\int_{\Omega}\left(\left|u^{\varepsilon, h}\right|^{2}+\left|e\left(u^{\varepsilon, h}\right)\right|^{2}\right) d \mu_{\varepsilon}^{h} \leqslant C<\infty
$$

(вытекающее из интегрального тождества (6.1) при $\varphi=u^{\varepsilon, h}$ в силу неравенства Корна (1.5)). Из него следуют сходимости (по крайней мере по подпоследовательности)

$$
\begin{gathered}
u^{\varepsilon, h} \rightarrow u^{\varepsilon} \quad \text { в } H^{1}(\Omega)^{2}, \quad e\left(u^{\varepsilon, h}\right) \rightarrow e\left(u^{\varepsilon}\right) \text { в } L^{2}(\Omega)^{3}, \\
\int_{\Omega} u^{\varepsilon, h} \cdot \varphi d \mu_{\varepsilon}^{h} \rightarrow \int_{\Omega} u^{\varepsilon} \cdot \varphi d \mu_{\varepsilon}, \\
\int_{\Omega} A e\left(u^{\varepsilon, h}\right) \cdot e(\varphi) d \lambda_{\varepsilon}^{h} \rightarrow \int_{\Omega} \widehat{A} e^{t}\left(u^{\varepsilon}\right) \cdot e^{t}(\varphi) d \lambda_{\varepsilon} \quad \forall \varphi \in C_{0}^{\infty}(\Omega)^{2},
\end{gathered}
$$


где $\widehat{A}$ - тензор релаксации, $e^{t}\left(u^{\varepsilon}\right)$ - тангенциальный градиент. Учитывая эти сходимости, приходим к тождеству (4.6), и тогда предельная функция $u^{\varepsilon}$, в самом деле, есть решение задачи (4.3). Подробнее аналогичный предельный переход при $h \rightarrow 0$, но в более простой ситуации был объяснен в $\S 3$.

В диаграммах (1.10) и (6.4) рассмотрим предельный переход “по диагонали", т.е. когда параметры $\varepsilon$ и $h$ зависимы, причем $h=h(\varepsilon) \rightarrow 0$ при $\varepsilon \rightarrow 0$.

Теорема 6.2. Для произвольной $h(\varepsilon) \rightarrow 0$ задачи (6.1) и (6.3) связаны сходимостью решений и энергий

$$
u^{\varepsilon, h} \rightarrow u \quad \text { в } \quad L^{2}(\Omega)^{2}, \quad J^{\varepsilon, h} \rightarrow J \quad \text { npu } \quad \varepsilon \rightarrow 0 .
$$

Эта теорема, так же как часть предыдушей, будет доказана позже, в конце 9.

\section{§7. Двухмасштабная сходимость с переменной составной мерой}

Изучим некоторую обшую ситуацию, в которую вписывается усреднение задач теории упругости на составных структурах, не обязательно плоских.

1. Пусть $\mu^{h}, \mu$ - 1-периодические борелевы меры в $\mathbb{R}^{d}$ такие, что $\mu^{h} \rightarrow \mu$ при $h \rightarrow 0$, и пусть $\mu_{\varepsilon}^{h}$ - масштабированная мера (см. $\left.\S 1\right)$, т.е. для фиксированного $h$

$$
\mu_{\varepsilon}^{h}(B)=\varepsilon^{d} \mu^{h}\left(\varepsilon^{-1} B\right) \text { для любого борелева множества } B \subset \mathbb{R}^{d} .
$$

Далее полагаем $h=h(\varepsilon) \rightarrow 0$ при $\varepsilon \rightarrow 0$. Тогда $d \mu_{\varepsilon}^{h} \rightarrow d x$ при $\varepsilon \rightarrow 0$.

Напомним, как определяется сходимость в переменном пространстве $L^{2}\left(\Omega, d \mu_{\varepsilon}^{h}\right)$ [1]-[3], [20].

Пусть $v^{\varepsilon, h}(x)$ ограничена в $L^{2}\left(\Omega, d \mu_{\varepsilon}^{h}\right)$, т.е. $\limsup _{\varepsilon \rightarrow 0} \int_{\Omega}\left|v^{\varepsilon, h}\right|^{2} d \mu_{\varepsilon}^{h}<\infty$.

Слабая сходимость $v^{\varepsilon, h} \rightarrow v$ в $L^{2}\left(\Omega, d \mu_{\varepsilon}^{h}\right)$ означает:

$$
v \in L^{2}(\Omega), \quad \lim _{\varepsilon \rightarrow 0} \int_{\Omega} v^{\varepsilon, h} \varphi d \mu_{\varepsilon}^{h}=\int_{\Omega} v \varphi d x \quad \forall \varphi \in C_{0}^{\infty}(\Omega) .
$$

Сильная сходимость $v^{\varepsilon, h} \rightarrow v$ в $L^{2}\left(\Omega, d \mu_{\varepsilon}^{h}\right)$ означает:

$$
\begin{gathered}
v \in L^{2}(\Omega), \lim _{\varepsilon \rightarrow 0} \int_{\Omega} v^{\varepsilon, h} z^{\varepsilon, h} d \mu_{\varepsilon}^{h}=\int_{\Omega} v z d x, \\
\text { как только } z^{\varepsilon, h} \rightarrow z \text { в } L^{2}\left(\Omega, d \mu_{\varepsilon}^{h}\right) .
\end{gathered}
$$

Сходимость в $L^{2}\left(\Omega, d \mu_{\varepsilon}^{h}\right)$ имеет следующие свойства:

1) всякая ограниченная в $L^{2}\left(\Omega, d \mu_{\varepsilon}^{h}\right)$ последовательность компактна в смысле слабой сходимости;

2) свойство полунепрерывности снизу: если $v^{\varepsilon, h} \rightarrow v$, то

$$
\liminf _{\varepsilon \rightarrow 0} \int_{\Omega}\left|v^{\varepsilon, h}\right|^{2} d \mu_{\varepsilon}^{h} \geqslant \int_{\Omega} v^{2} d x
$$

3) критерий компактности:

$$
v^{\varepsilon, h} \rightarrow v \Longleftrightarrow v^{\varepsilon, h} \rightarrow v \quad \text { и } \quad \lim _{\varepsilon \rightarrow 0} \int_{\Omega}\left|v^{\varepsilon, h}\right|^{2} d \mu_{\varepsilon}^{h}=\int_{\Omega} v^{2} d x .
$$


Мы будем использовать также двухмасштабную сходимость в пространстве $L^{2}\left(\Omega, d \mu_{\varepsilon}^{h}\right)[2],[3],[20]$, которая является обобщением двухмасштабной сходимости в $L^{2}(\Omega, d x)[21]$, [22].

Основу теории двухмасштабной сходимости составляет

Свойство среднего значения [3], [20]. Пусть $\Omega$-ограниченная измеримая по Жордану область и $a^{h} \rightarrow a$ в $L^{2}\left(Y, d \mu^{h}\right)$. Тогда для любого $\varphi \in C(\bar{\Omega})$

$$
\lim _{\varepsilon \rightarrow 0} \int_{\Omega} \varphi(x) a^{h}\left(\frac{x}{\varepsilon}\right) d \mu_{\varepsilon}^{h}=\int_{\Omega} \int_{Y} \varphi(x) a(y) d x d \mu .
$$

Дадим определение двухмасштабной сходимости в $L^{2}\left(\Omega, d \mu_{\varepsilon}^{h}\right)$.

Скажем, что ограниченная в $L^{2}\left(\Omega, d \mu_{\varepsilon}^{h}\right)$ последовательность $v^{\varepsilon, h}(x)$ cлабо двухмаситабно сходится к функции $v(x, y) \in L^{2}(\Omega \times Y, d x \times d \mu): v^{\varepsilon, h}(x) \stackrel{2}{\rightarrow} v(x, y)$, если

$$
\begin{gathered}
\lim _{\varepsilon \rightarrow 0} \int_{\Omega} v^{\varepsilon, h}(x) \varphi(x) b\left(\frac{x}{\varepsilon}\right) d \mu_{\varepsilon}^{h}=\int_{\Omega} \int_{Y} v(x, y) \varphi(x) b(y) d x d \mu \\
\forall \varphi \in C_{0}^{\infty}(\Omega), \quad \forall b \in C_{\text {per }}^{\infty}(Y) .
\end{gathered}
$$

Скажем, что последовательность $v^{\varepsilon, h}(x)$ сильно двухмасштабно сходится к функции $v(x, y) \in L^{2}(\Omega \times Y, d x \times d): v^{\varepsilon, h} \stackrel{2}{\rightarrow} v(x, y)$, если

$$
\begin{aligned}
\lim _{\varepsilon \rightarrow 0} \int_{\Omega} v^{\varepsilon, h}(x) z^{\varepsilon, h}(x) d \mu_{\varepsilon}^{h} \\
\quad=\int_{\Omega} \int_{Y} v(x, y) z(x, y) d x d \mu, \quad \text { как только } z^{\varepsilon, h}(x) \stackrel{2}{-} z(x, y) .
\end{aligned}
$$

Сходимости для вектор-функции $v^{\varepsilon, h}=\left(v_{1}^{\varepsilon, h}, \ldots, v_{N}^{\varepsilon, h}\right)$ определяются покомпонентно. Например, $v^{\varepsilon, h} \stackrel{2}{\rightarrow} v \Leftrightarrow v_{i}^{\varepsilon, h} \stackrel{2}{\rightarrow} v_{i}, i=1, \ldots, N$.

Перечислим некоторые обшие свойства двухмасштабной сходимости, которые используются далее.

(i) Всякая ограниченная в $L^{2}\left(\Omega, d \mu_{\varepsilon}^{h}\right)$ последовательность содержит слабо двухмасштабно сходяшуюся подпоследовательность.

(ii) Если $v^{\varepsilon, h}(x) \stackrel{2}{\rightarrow} v(x, y)$, то

$$
\liminf _{\varepsilon \rightarrow 0} \int_{\Omega}\left|v^{\varepsilon, h}\right|^{2} d \mu_{\varepsilon}^{h} \geqslant \int_{\Omega} \int_{Y}|v(x, y)|^{2} d x d \mu .
$$

(iii) $v^{\varepsilon, h}(x) \stackrel{2}{\rightarrow} v(x, y) \Leftrightarrow v^{\varepsilon, h}(x) \stackrel{2}{\rightarrow} v(x, y)$ и

$$
\lim _{\varepsilon \rightarrow 0} \int_{\Omega}\left|v^{\varepsilon, h}\right|^{2} d \mu_{\varepsilon}^{h}=\int_{\Omega} \int_{Y} v^{2}(x, y) d x d \mu .
$$

(iv) Если $v^{\varepsilon, h}(x) \stackrel{2}{\rightarrow} v(x, y)$, то $v^{\varepsilon, h}(x) \rightarrow \int_{Y} v(x, y) d \mu$. 
(v) Если $v^{\varepsilon, h}(x) \stackrel{2}{\rightarrow} v(x, y), b^{h}(y) \rightarrow b(y)$ в $L^{2}\left(Y, d \mu^{h}\right)$, то

$$
b^{h}\left(\frac{x}{\varepsilon}\right) v^{\varepsilon, h}(x) \stackrel{2}{-} b(y) v(x, y) .
$$

(vi) Пусть $v^{\varepsilon, h}(x) \stackrel{2}{\rightarrow} v(x, y)$, где $v(x, y)=\sum_{i} \varphi_{i}(x) b_{i}(y), \varphi_{i} \in C(\bar{\Omega}), b_{i} \in$ $L_{\mathrm{per}}^{2}(Y, d \mu)$, ипусть $b_{i}^{h} \rightarrow b_{i}$ в $L_{\mathrm{per}}^{2}\left(Y, d \mu^{h}\right), v^{h}(x, y)=\sum_{i} \varphi_{i}(x) b_{i}^{h}(y)$. Тогда

$$
\lim _{\varepsilon \rightarrow 0} \int_{\Omega}\left|v^{\varepsilon, h}(x)-v^{h}\left(x, \frac{x}{\varepsilon}\right)\right|^{2} d \mu_{\varepsilon}^{h}=0 .
$$

Приведем свойства сходимости для вектор-функций. В них участвует $A^{h}(y)-$ симметрическая 1-периодическая борелева матрица в $\mathbb{R}^{N}$ такая, что

$$
\begin{gathered}
c I_{N} \leqslant A^{h}(y) \leqslant c^{-1} I_{N}, \quad I_{N}-\text { единичная матрица в } \mathbb{R}^{N}, \quad c>0, \\
A^{h}(y) \rightarrow A(y) \text { в } L^{2}\left(Y, d \mu^{h}\right)^{N(N+1) / 2} .
\end{gathered}
$$

Пусть, далее, $A^{h}\left(\frac{x}{\varepsilon}\right)=A_{\varepsilon}^{h}(x)=A_{\varepsilon}^{h}$.

(vii) Если $v^{\varepsilon, h}(x) \stackrel{2}{\rightarrow} v(x, y)$ в $L^{2}\left(\Omega, d \mu_{\varepsilon}^{h}\right)^{N}$, то

$$
\liminf _{\varepsilon \rightarrow 0} \int_{\Omega} A^{h}\left(\frac{x}{\varepsilon}\right) v^{\varepsilon, h} \cdot v^{\varepsilon, h} d \mu_{\varepsilon}^{h} \geqslant \int_{\Omega} \int_{Y} A(y) v(x, y) \cdot v(x, y) d x d \mu
$$

причем это свойство остается в силе, если условие $A^{h}(y) \geqslant c I_{N}$ заменить на $A^{h}(y) \geqslant 0$.

(viii) Критерий сильной двухмасштабной сходимости векторов:

$$
\begin{gathered}
v^{\varepsilon, h}(x) \stackrel{2}{\rightarrow} v(x, y) \text { в } L^{2}\left(\Omega, d \mu_{\varepsilon}^{h}\right)^{N} \Longleftrightarrow v^{\varepsilon, h}(x) \stackrel{2}{\rightarrow} v(x, y) \text { в } L^{2}\left(\Omega, d \mu_{\varepsilon}^{h}\right)^{N}, \\
\lim _{\varepsilon \rightarrow 0} \int_{\Omega} A^{h}\left(\frac{x}{\varepsilon}\right) v^{\varepsilon, h}(x) \cdot v^{\varepsilon, h}(x) d \mu_{\varepsilon}^{h}=\int_{\Omega} \int_{Y} A(y) v(x, y) \cdot v(x, y) d x d \mu .
\end{gathered}
$$

Свойства (i)-(vi) доказаны в [3], [20]. Свойства (vii), (viii) - некоторые обобщения (например за счет зависимости матрицы $A_{\varepsilon}^{h}=A^{h}(x / \varepsilon)$ от двух параметров) аналогичных свойств, приведенных в [20] в теоремах 7.1, 7.2, и могут быть доказаны последовательно одно за другим с использованием свойства (v).

2. Сформулируем свойства двухмасштабной сходимости вектор-функций $u^{\varepsilon, h} \in$ $L^{2}\left(\Omega, d \mu_{\varepsilon}^{h}\right)^{d}$, которые обнаруживаются при совместном изучении $u^{\varepsilon, h}$ и $e\left(u^{\varepsilon, h}\right)$, если меры $\mu, \mu^{h}$ удовлетворяют следующим требованиям:

$$
\mathscr{R}(\mu)=\mathbb{R}^{d},
$$


т.е. пространство жестких периодических перемещений в $\mathscr{R}(\mu)$ состоит только из постоянных векторов;

$$
\begin{gathered}
\text { меры } \mu^{h}, \mu \text { связаны аппроксимативными условиями } \\
\quad \text { (см. определение } 5.3) ; \\
\text { мера } \mu \text { невырож дена, }
\end{gathered}
$$

т.е. $V_{\text {pot }}(Y, d \mu)$ не содержит постоянных матриц. Напомним, что $V_{\text {pot }}(Y, d \mu)$ есть замыкание множества $\left\{e(\phi), \phi \in C_{\text {per }}^{\infty}(Y)^{d}\right\}$ в $L^{2}(Y, d \mu)^{d(d+1) / 2}$.

условие (7.2) для пространства вектор-функций можно трактовать как некоторый аналог условия эргодичности меры в пространстве скалярных функций (см. $[1],[2])$.

Условие невырожденности меры (7.4) не сушественно для дальнейшего, оно лишь упрошает формулировку результатов и их доказательство. Невырожденность меры влечет невырожденность усредненного тензора $A^{\text {hom }}$, который в обшем случае лишш неотрицателен.

Далее $A^{h}(y)$ - 1-периодическая борелева симметрическая операторная функция, действуюшая в пространстве симметрических матриц $\mathbb{R}^{d(d+1) / 2}$ (тензор упругости), такая, что

$$
\begin{gathered}
c \xi \cdot \xi \leqslant A^{h}(y) \xi \cdot \xi \leqslant c^{-1} \xi \cdot \xi \quad \forall \xi \in \mathbb{R}^{d(d+1) / 2}, \quad c>0 \\
A^{h} \rightarrow A \text { в } L^{2}\left(Y, d \mu^{h}\right) .
\end{gathered}
$$

Пусть $A_{\varepsilon}^{h}=A_{\varepsilon}^{h}(x)=A^{h}(x / \varepsilon)$.

Для тензора $A$ из (7.6) усредненный тензор $A^{\text {hom }}$ можно определить по формуле

$$
A^{\mathrm{hom}} \xi \cdot \xi=\min _{v \in V_{\mathrm{pot}}(Y, d \mu)} \int_{Y} A(\xi+v) \cdot(\xi+v) d \mu
$$

Из (7.4), (7.5) вытекает невырожденность тензора $A^{\text {hom }}$.

Рассмотрим последовательность $u^{\varepsilon, h} \in C_{0}^{\infty}(\Omega)^{d}$, ограниченную вместес $e\left(u^{\varepsilon, h}\right)$ в $L^{2}\left(\Omega, d \mu_{\varepsilon}^{h}\right)$, т.е.

$$
\limsup _{\varepsilon \rightarrow 0} \int_{\Omega}\left(\left|u^{\varepsilon, h}\right|^{2}+\left|e\left(u^{\varepsilon, h}\right)\right|^{2}\right) d \mu_{\varepsilon}^{h}<\infty .
$$

Переходя, быть может, к подпоследовательности, будем считать, что

$$
u^{\varepsilon, h}(x) \stackrel{2}{\rightarrow} u(x, y), \quad e\left(u^{\varepsilon, h}\right) \stackrel{2}{\rightarrow} p(x, y) .
$$

В следующей теореме уточняются свойства этих сходимостей. 
TЕОРема 7.1. Пусть $h>0, h=h(\varepsilon) \rightarrow 0$ и выполнены условия (7.2)-(7.6). Тогда

$$
\begin{array}{ll}
u(x, y)=u(x) \in H_{0}^{1}(\Omega)^{d}, & u^{\varepsilon, h} \rightarrow u(x), \\
p(x, y)=e(u(x))+v(x, y), & v \in L^{2}\left(\Omega, V_{\mathrm{pot}}(Y, d \mu)\right) .
\end{array}
$$

Если дополнительно выполнено соотношение

$$
\lim _{\varepsilon \rightarrow 0} \int_{\Omega} A_{\varepsilon}^{h} e\left(u^{\varepsilon, h}\right) \cdot e_{y}\left(w\left(\frac{x}{\varepsilon}\right)\right) \varphi(x) d \mu_{\varepsilon}^{h}=0 \quad \forall \varphi \in C_{0}^{\infty}(\Omega), \quad \forall w \in C_{\mathrm{per}}^{\infty}(Y)^{d},
$$

mo

$$
A_{\varepsilon}^{h} e\left(u^{\varepsilon, h}\right) \rightarrow A^{\text {hom }} e(u)
$$

и в (7.11) $v(x, y)=\left.v_{\xi}(y)\right|_{\xi=е(u)}$, где $v_{\xi}(y)-$ минимизант задачи (7.7);

$$
\begin{aligned}
\liminf _{\varepsilon \rightarrow 0} \int_{\Omega} A_{\varepsilon}^{h} e\left(u^{\varepsilon, h}\right) \cdot e\left(u^{\varepsilon, h}\right) d \mu_{\varepsilon}^{h} & \geqslant \int_{\Omega} \int_{Y} A(e(u)+v) \cdot(e(u)+v) d x d \mu \\
& \geqslant \int_{\Omega} A^{\mathrm{hom}} e(u) \cdot e(u) d x
\end{aligned}
$$

Утверждения теоремь также верны, если всюду $h \equiv 0$ и $\mu_{\varepsilon}^{0}=\mu_{\varepsilon}, \mu_{\varepsilon}$ получена из $\mu$ по формуле (7.1), $A_{\varepsilon}^{0}=A_{\varepsilon}=A(x / \varepsilon)$.

Полное доказательство этой теоремы опускаем, так как оно во многом повторяет доказательства аналогичных утверждений для скалярных и векторных пространств из $[2 ; \S 4],[3 ; \S 9]$. Ключевыми в нашем доказательстве являются аппроксимативные условия для меры $\mu^{h}$ и

ЛЕММА ОБ АППРОКС ИМАцИИ [3; лемма 9.3]. Множество векторов $a=\operatorname{div} b$ (в смысле меры $\mu$ ) плотно в $\mathscr{R}(\mu)^{\perp}$.

Например покажем, как, используя эти моменты, выяснить структуру двухмасштабного предела из $(7.9)_{1}$, а именно $u(x, y)=u(x)$, даже при более общих предположениях, чем (7.8).

Справедливо следующее

УТвЕРЖДЕнИЕ. Пусть для $u^{\varepsilon, h} \in C^{\infty}(\Omega)^{d}$ выполнены условия:

$$
u^{\varepsilon, h}(x) \stackrel{2}{\rightarrow} u(x, y), \quad \varepsilon\left\|e\left(u^{\varepsilon, h}\right)\right\|_{L^{2}\left(\Omega, d \mu_{\varepsilon}^{h}\right)} \rightarrow 0 .
$$

Тогда $u(x, y)=u(x)$.

Для доказательства возьмем $a=\operatorname{div} b$ (в смысле меры $\mu$ ) и $a^{h}, b^{h}-$ из аппроксимативного свойства (i). Тогда из тождества (5.6) на периодических функциях следует аналогичное тождество на финитных функциях:

$$
-\varepsilon \int_{\Omega} b^{h}\left(\frac{x}{\varepsilon}\right) \cdot e(\psi) d \mu_{\varepsilon}^{h}=\int_{\Omega} a^{h}\left(\frac{x}{\varepsilon}\right) \cdot \psi d \mu_{\varepsilon}^{h}, \quad \psi \in C_{0}^{\infty}(\Omega)^{d} .
$$


Полагая $\psi=u^{\varepsilon, h} \phi, \phi \in C_{0}^{\infty}(\Omega)$, получаем

$-\varepsilon \int_{\Omega} \phi b^{h}\left(\frac{x}{\varepsilon}\right) \cdot e\left(u^{\varepsilon, h}\right) d \mu_{\varepsilon}^{h}=\int_{\Omega} \phi a^{h}\left(\frac{x}{\varepsilon}\right) \cdot u^{\varepsilon, h} d \mu_{\varepsilon}^{h}+\varepsilon \int_{\Omega}\left[u^{\varepsilon, h} \times \nabla \phi\right] \cdot b^{h}\left(\frac{x}{\varepsilon}\right) d \mu_{\varepsilon}^{h}$.

Отсюда, используя свойства функций $u^{\varepsilon, h}, a^{h}, b^{h}$, а также свойство (v) двухмасштабной сходимости, после перехода к пределу выводим

$$
\int_{\Omega} \int_{Y} u(x, y) \cdot \phi(x) a(y) d x d \mu=0
$$

что по лемме об аппроксимации в силу (7.2) дает искомое равенство $u(x, y)=u(x)$.

3. Свяжем с ограниченной липшицевой областью $\Omega$ пространство $\mathscr{H}^{\varepsilon, h}$-замыкание множества $C_{0}^{\infty}(\Omega)^{d}$ по норме $\left(\int_{\Omega}\left(|\varphi|^{2}+|e(\varphi)|^{2}\right) d \mu_{\varepsilon}^{h}\right)^{1 / 2}$. Изучим следующую задачу теории упругости:

$u^{\varepsilon, h} \in \mathscr{H}^{\varepsilon, h}, \quad \int_{\Omega}\left(A_{\varepsilon}^{h} e\left(u^{\varepsilon, h}\right) \cdot e(\varphi)+u^{\varepsilon, h} \cdot \varphi\right) d \mu_{\varepsilon}^{h}=\int_{\Omega} f^{\varepsilon, h} \cdot \varphi d \mu_{\varepsilon}^{h} \quad \forall \varphi \in C_{0}^{\infty}(\Omega)^{d}$,

где $f^{\varepsilon, h}$ ограничены в $L^{2}\left(\Omega, d \mu_{\varepsilon}^{h}\right)^{d}$, тензор $A_{\varepsilon}^{h}=A^{h}(x / \varepsilon)$ удовлетворяет условиям $(7.5),(7.6)$.

Существование и единственность решения как пары $u^{\varepsilon, h}, e\left(u^{\varepsilon, h}\right)$ следует из теоремы Рисса.

Введем усредненную задачу

$$
u \in H_{0}^{1}(\Omega)^{d}, \quad \int_{\Omega}\left(A^{\mathrm{hom}} e(u) \cdot e(\varphi)+u \cdot \varphi\right) d x=\int_{\Omega} f \cdot \varphi d x \quad \forall \varphi \in C_{0}^{\infty}(\Omega)^{d} .
$$

Очевидны существование и единственность этой задачи в силу постоянства и невырожденности тензора $A^{\text {hom }}$.

Связь меж ду решениями задач (7.15), (7.16) устанавливает следуюшая

Теорема 7.2. Пусть $h>0, h(\varepsilon) \rightarrow 0$ и выполнены условия (7.2)-(7.6). Тогда если

$$
f^{\varepsilon, h}(x) \rightarrow f(x) \quad \text { в } \quad L^{2}\left(\Omega, d \mu_{\varepsilon}^{h}\right)^{d},
$$

mo

$$
\begin{aligned}
& u^{\varepsilon, h}(x) \rightarrow u(x) \quad \text { в } L^{2}\left(\Omega, d \mu_{\varepsilon}^{h}\right)^{d}, \\
& e\left(u^{\varepsilon, h}(x)\right) \stackrel{2}{\rightarrow} e(u(x))+v(x, y) \quad \boldsymbol{\theta} \quad L^{2}\left(\Omega, d \mu_{\varepsilon}^{h}\right)^{d(d+1) / 2},
\end{aligned}
$$

2дe

$$
\begin{gathered}
v(x, y)=\left.v_{\xi}(y)\right|_{\xi=e(u(x))}, \quad v_{\xi}(\cdot)-\text { минимизант задачи }(7.7), \\
\text { m.е. } \quad \operatorname{div}_{y} A(e(u(x))+v(x, y))=0 \quad(\text { в смиьсле меры } \mu) .
\end{gathered}
$$

Если сходимость правых частей (7.17) является сильной, то сильными будут и сходимости решений и их градиентов в (7.18) и (7.19). При этом имеет место сходимость әнергий

$$
\lim _{\varepsilon \rightarrow 0} \int_{\Omega} A_{\varepsilon}^{h} e\left(u^{\varepsilon, h}\right) \cdot e\left(u^{\varepsilon, h}\right) d \mu_{\varepsilon}^{h}=\int_{\Omega} A^{\mathrm{hom}} e(u) \cdot e(u) d x .
$$

Утверждения теоремы верны также при $h \equiv 0, к о г д а ~ \mu_{\varepsilon}^{0}=\mu_{\varepsilon}$. 
ДокАЗАТЕЛЬСтво. Из интегрального тождества (7.15) с $\varphi=u^{\varepsilon, h}$ следует условие ограниченности (7.8), благодаря которому, переходя, быть может, к подпоследовательности (см. свойство (i) двухмасштабной сходимости), считаем выполненными соотношения (7.9), (7.10).

Взяв в тождестве (7.15) пробную функцию вида $\varphi(x)=\varepsilon \psi(x) w\left(\varepsilon^{-1} x\right), \psi \in$ $C_{0}^{\infty}(\Omega), w \in C_{\mathrm{per}}^{\infty}(Y)^{d}$, после перехода к пределу получим условие (7.12), из которого вытекает сходимость (7.13). Тогда можно перейти к пределу в тож дестве (7.15) с произвольной функцией $\varphi \in C_{0}^{\infty}(\Omega)$, в результате чего получим тождество (7.16) для предельной функции $u$. В силу единственности решения задачи (7.16) сходимость (7.18) доказана для всей последовательности $\varepsilon$.

Пусть в (7.17) имеем сильную сходимость $f^{\varepsilon, h} \rightarrow f$. Сравним энергетические равенства для задач $(7.15),(7.16)$ :

$$
\begin{aligned}
\int_{\Omega}\left[A_{\varepsilon}^{h} e\left(u^{\varepsilon h}\right) \cdot e\left(u^{\varepsilon, h}\right)+u^{\varepsilon, h} \cdot u^{\varepsilon, h}\right] d \mu_{\varepsilon}^{h} & =\int_{\Omega} f^{\varepsilon, h} \cdot u^{\varepsilon, h} d \mu_{\varepsilon}^{h}, \\
\int_{\Omega}\left[A_{\varepsilon}^{\mathrm{hom}} e(u) \cdot e(u)+u \cdot u\right] d x & =\int_{\Omega} f \cdot u d x .
\end{aligned}
$$

Поскольку слабая сходимость (7.18) уже доказана, то по определению сильной сходимости $\int_{\Omega} f^{\varepsilon, h} \cdot u^{\varepsilon, h} d \mu_{\varepsilon}^{h} \rightarrow \int_{\Omega} f \cdot u d x$. Следовательно,

$$
\lim _{\varepsilon \rightarrow 0} \int_{\Omega}\left[A_{\varepsilon}^{h} e\left(u^{\varepsilon, h}\right) \cdot e\left(u^{\varepsilon, h}\right)+u^{\varepsilon, h} \cdot u^{\varepsilon, h}\right] d \mu_{\varepsilon}^{h}=\int_{\Omega}\left[A^{\text {hom }} e(u) \cdot e(u)+u \cdot u\right] d x .
$$

Из свойств полунепрерывности для двухмасштабной сходимости (см. (ii) и (vii)) выводим отсюда сходимость энергий (7.21), а также сходимость норм

$$
\int_{\Omega}\left|u^{\varepsilon, h}\right|^{2} d \mu_{\varepsilon}^{h} \rightarrow \int_{\Omega}|u|^{2} d x
$$

Из сходимости энергий (7.21) следует, что в соотношениях (7.14) для решения $u^{\varepsilon, h}$ всюду имеют место равенства. В частности,

$$
\liminf _{\varepsilon \rightarrow 0} \int_{\Omega} A_{\varepsilon}^{h} e\left(u^{\varepsilon, h}\right) \cdot e\left(u^{\varepsilon, h}\right) d \mu_{\varepsilon}^{h}=\int_{\Omega} \int_{Y} A(e(u)+v) \cdot(e(u)+v) d x d \mu .
$$

Отсюда в силу критериев сильной сходимости (iii), (viii) получаем сильную сходимость решений и их градиентов. Теорема доказана.

В следующей теореме мера $\mu^{h}$ удовлетворяет дополнительному требованию, а именно пусть выполнено равномерное (по $h$ ) неравенство Корна для периодических функцций

$$
\int_{Y}|\varphi|^{2} d \mu^{h} \leqslant c \int_{Y}|e(\varphi)|^{2} d \mu^{h} \forall \varphi \in C_{\mathrm{per}}^{\infty}(Y)^{d}, \quad \int_{Y} \varphi d \mu^{h}=0 .
$$


Изнего, в частности, вытекает возможность представить матрицу $v \in V_{\text {pot }}\left(Y, d \mu^{h}\right)$ в виде $v=e(w), w \in H_{\mathrm{per}}^{1}\left(Y, d \mu^{h}\right)^{d}$. Поэтому и минимизант вариационной задачи

$$
A_{h}^{\text {hom }} \xi \cdot \xi=\min _{v \in V_{\text {pot }}\left(Y, d \mu^{h}\right)} \int_{Y} A^{h}(\xi+v) \cdot(\xi+v) d \mu^{h}
$$

допускает представление $v_{\xi}^{h}(y)=e_{y}\left(w^{h}(\xi, y)\right)$, где

$$
\begin{gathered}
\left.w^{h}(\xi, y) \in H_{\mathrm{per}}^{1}\left(Y, d \mu^{h}\right)^{d}, \quad \operatorname{div}_{y} A^{h}\left(\xi+e_{y}\left(w^{h}\right)\right)=0 \quad \text { (в смысле меры } \mu^{h}\right), \\
\int_{Y} w^{h}(\xi, \cdot) d \mu^{h}=0 .
\end{gathered}
$$

TЕОрема 7.3 (окорректоре). Пусть в предположениях теоремы $7.2 f^{\varepsilon, h} \rightarrow f$ в $L^{2}\left(\Omega, d \mu_{\varepsilon}^{h}\right)^{d}, u \in C^{2}(\bar{\Omega})^{d}$. Тогда если $u_{1}(x, y)=w^{h}(e(u(x)), y), w^{h}(\xi, y)$ из (7.24), mо

$$
\lim _{\varepsilon \rightarrow 0} \int_{\Omega}\left|e\left(u^{\varepsilon, h}(x)-u(x)-\varepsilon u_{1}\left(x, \frac{x}{\varepsilon}\right)\right)\right|^{2} d \mu_{\varepsilon}^{h}=0 .
$$

ДокАЗАТЕЛЬСТво. По теореме 7.2

$$
e\left(u^{\varepsilon, h}(x)\right) \stackrel{2}{\rightarrow} e(u(x))+v(x, y)=p(x, y) .
$$

Теперь докажем сходимость

$$
v_{\xi}^{h}(y) \rightarrow v_{\xi}(y) \quad \text { в } L^{2}\left(Y, d \mu^{h}\right)^{d}
$$

для минимизантов задач (7.23) и (7.7). Из интегрального тождества для $v_{\xi}^{h}=v^{h}$ :

$$
v^{h} \in V_{\mathrm{pot}}\left(Y, d \mu^{h}\right), \quad \int_{Y} A^{h}\left(\xi+v^{h}\right) \cdot e(\varphi) d \mu^{h}=0 \quad \forall \varphi \in C_{\mathrm{per}}^{\infty}(Y)^{d},
$$

вытекает оценка $\int_{Y} A^{h} v^{h} \cdot v^{h} d \mu^{h} \leqslant \int_{Y} A^{h} \xi \cdot \xi d \mu^{h}$, поэтому можно считать, что $v^{h} \rightarrow v$. Аппроксимативные свойства меры $\mu^{h}$ обеспечивают [3; §16] свойство сохранения потенциальности при слабой сходимости матриц. Тогда переход к пределу в (7.28) дает (см. (7.6))

$$
v \in V_{\mathrm{pot}}(Y, d \mu), \quad \int_{Y} A(\xi+v) \cdot e(\varphi) d \mu=0 \quad \forall \varphi \in C_{\mathrm{per}}^{\infty}(Y)^{d},
$$

и, действительно, $v=v_{\xi}$ - минимизант задачи (7.7).

С помошью энергетических равенств для $v_{\xi}^{h}=v^{h}, v_{\xi}=v$ выводим сходимость квадратических форм

$$
\int_{Y} A^{h} v^{h} \cdot v^{h} d \mu^{h}=-\int_{Y} A^{h} \xi \cdot v^{h} d \mu^{h} \rightarrow-\int_{Y} A \xi \cdot v d \mu=\int_{Y} A v \cdot v d \mu,
$$


что вместе со слабой сходимостью $v^{h} \rightarrow v$ влечет (7.27) согласно критерию сильной сходимости векторов (матриц) в $L^{2}\left(Y, d \mu^{h}\right)$ (см. выше аналогичный критерий (viii) сильной двухмасштабной сходимости).

Нетрудно увидеть, что функция $v(x, y)$ из (7.20) имеет структуру, предусмотренную свойством (vi) двухмасштабной сходимости. Положим $p^{h}(x, y)=e(u(x))+$ $v^{h}(x, y)$, где $v^{h}(x, y)=\left.v_{\xi}^{h}(y)\right|_{\xi=e(u(x))}$. Тогда из $(7.26),(7.27)$ следует

$$
\lim _{\varepsilon \rightarrow 0} \int_{\Omega}\left|e\left(u^{\varepsilon, h}\right)-p^{h}\left(x, \frac{x}{\varepsilon}\right)\right|^{2} d \mu_{\varepsilon}^{h}=0
$$

что в силу представления $v^{h}(x, y)=e_{y}\left(w^{h}(x, y)\right)$ с учетом неравенства (7.22) дает соотношения (7.25).

\section{§8. Усреднение на составной структуре}

Применим результаты $\S 7$ к усреднению на составной структуре. Следуюшая теорема суммирует результаты $\S 5$, благодаря которым это можно сделать.

Tеорема 8.1. Для составных мер $\mu, \mu^{h}$, заданных в (1.1), выполнены условия (7.2)-(7.4).

Вместо (6.1) рассмотрим более общую задачу Дирихле в статической постановке с переменным тензором упругости и переменной правой частью:

$$
u^{\varepsilon, h} \in H_{0}^{1}(\Omega)^{2}, \quad \int_{\Omega} A_{\varepsilon}^{h} e\left(u^{\varepsilon, h}\right) \cdot e(\varphi) d \mu_{\varepsilon}^{h}=\int_{\Omega} f^{\varepsilon, h} \cdot \varphi d \mu_{\varepsilon}^{h} \quad \forall \varphi \in C_{0}^{\infty}(\Omega)^{2},
$$

где $A_{\varepsilon}^{h}=A_{\varepsilon}^{h}(x)=A^{h}(x / \varepsilon), A^{h}(y)$ - семейство 1-периодических тензоров упругости, удовлетворяющих условиям (7.5), (7.6), а правая часть $f^{\varepsilon, h} \in L^{2}\left(\Omega, d \mu_{\varepsilon}^{h}\right)$ и ограничена в $L^{2}\left(\Omega, d \mu_{\varepsilon}^{h}\right)^{2}$. Как и раншше, допускаем $h \equiv 0$.

Разрешимость задачи (8.1), а также условие равномерной ограниченности (7.8) вытекают из предположений на тензор $A_{\varepsilon}^{h}$ и правую часть $f^{\varepsilon, h}$ в силу неравенства Корна (1.5).

Теорема 8.2. Пусть $f^{\varepsilon, h}(x) \rightarrow f(x)$ в $L^{2}\left(\Omega, d \mu_{\varepsilon}^{h}\right)^{2}$. Тогда для решений задачи (8.1) имеет место сходимость (7.18), где предельная функиия и( $(x)$ есть решение задачи (6.3).

Пусть

$$
f^{\varepsilon, h}(x) \rightarrow f(x) \quad \text { в } \quad L^{2}\left(\Omega, d \mu_{\varepsilon}^{h}\right)^{2},
$$

тогда и сходимость решений будет сильной, при этом имеет место сходимость упругих энергий (7.21).

Пусть $u(x) \in C^{2}(\bar{\Omega})^{2}$, тогда имеет место сходимость с корректором вида (7.25). 
ДокАЗАТЕЛЬСТво. Без потери общности считаем, что

$$
u^{\varepsilon, h}(x) \rightarrow u(x) \text { в } L^{2}\left(\Omega, d \mu_{\varepsilon}^{h}\right)^{2},
$$

причем $u(x) \in H_{0}^{1}(\Omega)^{2}$ (см. теорему 7.1). Запишем задачу (8.1) в резольвентной форме (7.15), а именно:

$$
u^{\varepsilon, h} \in \mathscr{H}^{\varepsilon, h}, \quad \int_{\Omega}\left(A_{\varepsilon}^{h} e\left(u^{\varepsilon, h}\right) \cdot e(\varphi)+u^{\varepsilon, h} \cdot \varphi\right) d \mu_{\varepsilon}^{h}=\int_{\Omega} g^{\varepsilon, h} \cdot \varphi d \mu_{\varepsilon}^{h},
$$

где $g^{\varepsilon, h}=u^{\varepsilon, h}+f^{\varepsilon, h}$ и $\mathscr{H}^{\varepsilon, h}=H_{0}^{1}(\Omega)^{2}$ в силу составной структуры меры $\mu^{h}$ из (1.1). Поскольку $g^{\varepsilon, h}(x) \rightarrow g(x)=u(x)+f(x)$, то по теореме 7.2 предельная функция $u$ есть решение усредненной задачи (7.16) с правой частью $g$, а значит, и задачи (5.3). Этим исчерпан случай слабой сходимости правых частей.

В предположении (8.2) сходимость энергий устанавливается так же, как в теореме 7.2. Докажем сильную сходимость решений. Для этого рассмотрим второй вариант задачи (8.1):

$$
v^{\varepsilon, h} \in H_{0}^{1}(\Omega)^{2}, \quad \int_{\Omega} A_{h}^{\varepsilon} e\left(v^{\varepsilon, h}\right) \cdot e(\varphi) d \mu_{\varepsilon}^{h}=\int_{\Omega} z^{\varepsilon, h} \cdot \varphi d \mu_{\varepsilon}^{h} \quad \forall \varphi \in C_{0}^{\infty}(\Omega),
$$

где в правой части стоит произвольная вектор-функция $z^{\varepsilon, h} \rightarrow z$. Тогда $v^{\varepsilon, h} \rightarrow v$, где $v$ - решение усредненного уравнения (6.3) с правой частью $z$.

Полагая в (8.3) $\varphi=u^{\varepsilon, h}$, а в (8.1) $\varphi=v^{\varepsilon, h}$, получаем по формуле Грина-Бетти равенство

$$
\int_{\Omega} u^{\varepsilon, h} \cdot z^{\varepsilon, h} d \mu_{\varepsilon}^{h}=\int_{\Omega} f^{\varepsilon, h} \cdot v^{\varepsilon, h} d \mu_{\varepsilon}^{h}
$$

правая часть которого по определению сильной сходимости $\left(f^{\varepsilon, h} \rightarrow f, v^{\varepsilon, h} \rightarrow v\right)$ сходится $\mathrm{K}$

$$
\int_{\Omega} f \cdot v d x=\int_{\Omega} u \cdot z d x
$$

Последнее равенство вьводится из интегрального тождества для предельных функций $u, v$. В результате

$$
\int_{\Omega} u^{\varepsilon, h} \cdot z^{\varepsilon, h} d \mu_{\varepsilon}^{h} \rightarrow \int_{\Omega} u \cdot z d x
$$

что в силу произвольности $z^{\varepsilon, h} \rightarrow z$ означает искомую сильную сходимость решений.

Искомая сходимость с корректором вытекает из теоремы 7.3 и доказанного в $\S 9$ неравенства Корна (7.22) с составной мерой $\mu^{h}$ (см. (9.1)). 


\section{§9. Неравенства Пуанкаре и Корна для составной меры. Принцип компактности}

1. Пусть $\lambda, \lambda^{h}, \mu, \mu^{h}$ - меры из (1.1), $\Omega$ - ограниченная липшицева область.

Лемма 9.1. $1^{\circ}$. Для периодических функиий имеют место равномерные неравенства Корна и Пуанкаре при $h \geqslant 0$ :

$$
\begin{gathered}
\int_{Y}|u|^{2} d \mu^{h} \leqslant C_{1} \int_{Y}|e(u)|^{2} d x, \quad u \in C_{\mathrm{per}}^{\infty}(Y)^{2}, \\
\int_{Y} u d \mu^{h}=0, \quad C_{1}=\operatorname{const}(F), \\
\int_{Y} v^{2} d \mu^{h} \leqslant C_{2} \int_{Y}|\nabla v|^{2} d x, \quad v \in C_{\mathrm{per}}^{\infty}(Y), \\
\int_{Y} v d \mu^{h}=0, \quad C_{2}=\operatorname{const}(F) .
\end{gathered}
$$

$2^{\circ}$. Для финитных функиий имеют место неравенство Корна (1.5), а также следующие равномерные (по $\varepsilon$ и $h \geqslant 0)$ неравенства:

$$
\begin{array}{ll}
\int_{\Omega} v^{2} d \mu_{\varepsilon}^{h} \leqslant C \int_{\Omega}|\nabla v|^{2} d x, & v \in C_{0}^{\infty}(\Omega), C=\operatorname{const}(\Omega, F), \\
\int_{\mathbb{R}^{2}} v^{2} d \lambda_{\varepsilon}^{h} \leqslant C \int_{\mathbb{R}^{2}}\left(v^{2}+\varepsilon^{2}|\nabla v|^{2}\right) d x, & v \in C_{0}^{\infty}\left(\mathbb{R}^{2}\right), C=\operatorname{const}(F) .
\end{array}
$$

ДокАЗАТЕЛЬСТво. Полезны следуюшие известные или легко выводимые неравенства:

$$
\begin{array}{rlrl}
\int_{Y} \varphi^{2} d \lambda^{h} & \leqslant C_{0} \int_{Y}\left(\varphi^{2}+|\nabla \varphi|^{2}\right) d x, & \varphi \in C^{\infty}(\bar{Y}), C_{0}=\operatorname{const}(F), h \geqslant 0, \\
\int_{\Omega} v^{2} d x \leqslant C \int_{\Omega}|\nabla v|^{2} d x, & & v \in C_{0}^{\infty}(\Omega), \quad C=\operatorname{const}(\Omega), \\
\int_{\Omega}|\nabla u|^{2} d x & \leqslant 2 \int_{\Omega}|e(u)|^{2} d x, & & u \in C_{0}^{\infty}(\Omega)^{2} .
\end{array}
$$

Гомотетическое сжатие переводит неравенство (9.5) в неравенство для квадрата $\varepsilon Y$

$$
\int_{\varepsilon Y} \varphi^{2} d \lambda_{\varepsilon}^{h} \leqslant C_{0} \int_{\varepsilon Y}\left(\varphi^{2}+\varepsilon^{2}|\nabla \varphi|^{2}\right) d x, \quad \varphi \in C^{\infty}(\varepsilon \bar{Y}),
$$

из которого, разбивая плоскость на непересекаюшиеся полуоткрытые квадраты $\mathbb{R}^{2}=\bigcup_{i} \varepsilon Y_{i}$, получаем неравенство (9.4):

$$
\begin{aligned}
\int_{\mathbb{R}^{2}} v^{2} d \lambda_{\varepsilon}^{h} & =\sum_{i} \int_{\varepsilon Y_{i}} v^{2} d \lambda_{\varepsilon}^{h} \leqslant C_{0} \sum_{i} \int_{\varepsilon Y_{i}}\left(v^{2}+\varepsilon^{2}|\nabla v|^{2}\right) d x \\
& \leqslant C_{0} \int_{\mathbb{R}^{2}}\left(v^{2}+\varepsilon^{2}|\nabla v|^{2}\right) d x .
\end{aligned}
$$

Отсюда и из $(9.6),(9.7)$ выводим последовательно неравенства (9.3) и (1.5).

Неравенства для периодических функций доказываются аналогично. 
2. Докажем с помошью неравенства (9.4) некоторые специфические свойства сходимости в пространстве $L^{2}\left(\Omega, d \mu_{\varepsilon}^{h}\right)$ с составной мерой $\mu_{\varepsilon}^{h}$.

ЛЕмма 9.2. Имеет место сходимость

$$
\lim _{\varepsilon \rightarrow \infty} \int_{\Omega} v^{2} d \lambda_{\varepsilon}^{h}=\int_{\Omega} v^{2} d x, \quad v \in H_{0}^{1}(\Omega) .
$$

ДокАЗАТЕЛЬСтво. Для $v \in C_{0}^{\infty}(\Omega)$ равенство $(9.8)$ следует из слабой сходимости мер $d \lambda_{\varepsilon}^{h} \rightarrow d x$. Для произвольной $v \in H_{0}^{1}(\Omega)$ имеем оценку

$$
\begin{aligned}
\left|\int_{\Omega} v^{2} d \lambda_{\varepsilon}^{h}-\int_{\Omega} v^{2} d x\right| \leqslant & \int_{\Omega}\left|v-v_{\delta}\right|^{2} d \lambda_{\varepsilon}^{h}+\left|\int_{\Omega} v_{\delta}^{2} d \lambda_{\varepsilon}^{h}-\int_{\Omega} v_{\delta}^{2} d x\right| \\
& +\left|\int_{\Omega} v_{\delta}^{2} d x-\int_{\Omega} v^{2} d x\right|
\end{aligned}
$$

в которой используем

$$
v_{\delta} \in C_{0}^{\infty}(\Omega), \quad\left|v-v_{\delta}\right|_{H^{1}(\Omega)}^{2}<\delta,
$$

и, значит, в силу (9.3)

$$
\int_{\Omega}\left|v-v_{\delta}\right|^{2} d \lambda_{\varepsilon}^{h}<C_{0} \delta
$$

Поэтому

$$
\limsup _{\varepsilon \rightarrow 0}\left|\int_{\Omega} v^{2} d \lambda_{\varepsilon}^{h}-\int_{\Omega} v^{2} d x\right| \leqslant C \delta
$$

где $\delta$ любое, а константа $C$ не зависит от $\delta$. Отсюда следует формула (9.8). Лемма доказана.

ЛЕмма 9.3. Пусть $v_{\varepsilon}$ ограничена в $H_{0}^{1}(\Omega)$. Тогда

$$
\text { если } \quad v_{\varepsilon} \rightarrow v \quad \text { в } L^{2}(\Omega), \quad \text { mo } \quad v_{\varepsilon} \rightarrow v \quad \text { в } \quad L^{2}\left(\Omega, d \mu_{\varepsilon}^{h}\right) .
$$

ДокАЗАТЕЛЬСТво. Необходимо доказать сходимость $v_{\varepsilon} \rightarrow v$ в $L^{2}\left(\Omega, d \lambda_{\varepsilon}^{h}\right)$, которая согласно свойствам сходимости в $L^{2}\left(\Omega, d \lambda_{\varepsilon}^{h}\right)$ (см. в $\S 7$ критерий сильной сходимости и свойство полунепрерывности снизу) выводится из соотношений

$$
\begin{aligned}
& \lim _{\varepsilon \rightarrow 0} \int_{\Omega} v_{\varepsilon} \varphi d \lambda_{\varepsilon}^{h}=\int_{\Omega} v \varphi d x \quad \forall \varphi \in C_{0}^{\infty}(\Omega), \\
& \lim _{\varepsilon \rightarrow 0} \int_{Y}\left|v_{\varepsilon}\right|^{2} d \lambda_{\varepsilon}^{h} \leqslant \int_{Y} v^{2} d x .
\end{aligned}
$$

Для $v_{\delta}$ из $(9.9)$ имеет место представление

$$
\begin{aligned}
\int_{\Omega} v_{\varepsilon} \varphi d \lambda_{\varepsilon}^{h}-\int_{\Omega} v \varphi d x= & \int_{\Omega}\left(v_{\varepsilon}-v\right) \varphi d \lambda_{\varepsilon}^{h}+\int_{\Omega}\left(v-v_{\delta}\right) \varphi d \lambda_{\varepsilon}^{h} \\
& +\left(\int_{\Omega} v_{\delta} \varphi d \lambda_{\varepsilon}^{h}-\int_{\Omega} v_{\delta} \varphi d x\right)+\int_{\Omega}\left(v_{\delta}-v\right) \varphi d x .
\end{aligned}
$$


В последней сумме второе и четвертое слагаемые можно оценить, используя $(9.9)_{2}$ и (9.4):

$$
\left|\int_{\Omega}\left(v-v_{\delta}\right) \varphi d \lambda_{\varepsilon}^{h}\right| \leqslant C_{\varphi} \delta, \quad\left|\int_{\Omega}\left(v_{\delta}-v\right) \varphi d x\right| \leqslant C_{\varphi} \delta .
$$

Там же первое и третье слагаемые в пределе при $\varepsilon \rightarrow 0$ дают нуль, так как

$$
\begin{aligned}
\int_{\Omega}\left|v_{\varepsilon}-v\right|^{2} d \lambda_{\varepsilon}^{h} & \leqslant C_{0} \int_{\Omega}\left(\left|v_{\varepsilon}-v\right|^{2}+\varepsilon^{2}\left|\nabla\left(v_{\varepsilon}-v\right)\right|^{2}\right) d x \\
& =C_{0} \int_{\Omega}\left|v_{\varepsilon}-v\right|^{2} d x+O\left(\varepsilon^{2}\right)=o(1)
\end{aligned}
$$

в силу (9.4) и $(9.10)_{1}$, а вследствие сходимости мер $d \lambda_{\varepsilon}^{h} \rightarrow d x$

$$
\int_{\Omega} v_{\delta} \varphi d \lambda_{\varepsilon}^{h} \rightarrow \int_{\Omega} v_{\delta} \varphi d x
$$

Поэтому

$$
\limsup _{\varepsilon \rightarrow 0}\left(\int_{\Omega} v_{\varepsilon} \varphi d \lambda_{\varepsilon}^{h}-\int_{\Omega} v \varphi d x\right) \leqslant C_{\varphi} \delta
$$

откуда в силу произвольности $\delta$ вытекает (9.11) 1 .

Соотношение $(9.11)_{2}$ следует из (9.12) и (9.8):

$$
\lim _{\varepsilon \rightarrow 0} \int_{\Omega}\left|v_{\varepsilon}\right|^{2} d \lambda_{\varepsilon}^{h} \leqslant \lim _{\varepsilon \rightarrow 0} \int_{\Omega}\left|v_{\varepsilon}-v\right|^{2} d \lambda_{\varepsilon}^{h}+\lim _{\varepsilon \rightarrow 0} \int_{\Omega} v^{2} d \lambda_{\varepsilon}^{h}=\int_{\Omega} v^{2} d x .
$$

Лемма доказана.

ТЕОРема 9.4 (принцип компактности).

$1^{\circ}$. Последовательность функций $v^{\varepsilon, h} \in C_{0}^{\infty}(\Omega)$ таких, что

$$
v^{\varepsilon, h}, \quad \nabla v^{\varepsilon, h} \quad \text { ограничены в } L^{2}(\Omega, d x),
$$

компактна в смицсле сильной сходимости в $L^{2}\left(\Omega, d \mu_{\varepsilon}^{h}\right)$.

$2^{\circ}$. Последовательность вектор-функиий $u^{\varepsilon, h} \in C_{0}^{\infty}(\Omega)^{2}$ таких, что

$$
\left.u^{\varepsilon, h}, \quad \text { e(u } u^{\varepsilon, h}\right) \quad \text { ограничены в } L^{2}(\Omega, d x),
$$

компактна в смысле сильной сходимости в $L^{2}\left(\Omega, d \mu_{\varepsilon}^{h}\right)^{2}$.

Простьм следствием из леммы 9.3 является первое утверждение этой теоремы. Из него вытекает второе утверждение, поскольку в силу неравенства Корна (9.7) условие (9.14) влечет условие (9.13) для каждой компоненты вектора $u^{\varepsilon, h}$.

Лемма 9.5. Утверждения лемм 9.2 и 9.3 остаются в силе для функций uз $H^{1}(\Omega)$. 
ДокАЗАТЕЛЬСТво. Из слабой сходимости мер $d \mu_{\varepsilon}^{h} \rightarrow d x$ (см. определение в 1 ) следует сходимость [20; лемма 1.1]

$$
\lim _{\varepsilon \rightarrow 0} \int_{\Omega} \varphi d \mu_{\varepsilon}^{h}=\int_{\Omega} \varphi d x, \quad \varphi \in C(\bar{\Omega}) .
$$

Отсюда ясно, что для вывода (9.8) в случае $v \in H^{1}(\Omega)$ рассматриваем в $(9.9)$ $v_{\delta} \in C^{\infty}(\bar{\Omega})$ и в остальном повторяем прежнее доказательство. Лемма доказана.

ЛЕмма 9.6. Если $v_{\varepsilon} \rightarrow v$ в $L^{2}\left(\Omega, d \mu_{\varepsilon}^{h}\right), v \in H^{1}(\Omega)$, mo

$$
\lim _{\varepsilon \rightarrow 0} \int_{\Omega}\left|v_{\varepsilon}-v\right|^{2} d \mu_{\varepsilon}^{h} \rightarrow 0
$$

ДокАЗАТЕльСтво. Заметим, что если $v \in H^{1}(\Omega)$, то $v \rightarrow v$ в $L^{2}\left(\Omega, d \mu_{\varepsilon}^{h}\right.$ ) (для вывода возьмем $v_{\varepsilon}=v$ и воспользуемся леммой 9.5$)$ и, значит, $\left(v_{\varepsilon}-v\right) \rightarrow 0$ в $L^{2}\left(\Omega, d \mu_{\varepsilon}^{h}\right)$, что по критерию сильной сходимости влечет (9.15).

ДокаЗАТЕльСтво теоремы 6.2. Для $f \in C^{\infty}(\bar{\Omega})^{2}$ имеем $f^{\varepsilon, h} \equiv f \rightarrow f$ в $L^{2}\left(\Omega, d \mu_{\varepsilon}^{h}\right)^{2}$ (см. вывод (9.15)). Отсюда по теореме 8.2 получаем сходимость энергий $(6.5)_{2}$, а также сильную сходимость решений в $L^{2}\left(\Omega, d \mu_{\varepsilon}^{h}\right)^{2}$, из которой по лемме 9.6 следует $(6.5)_{1}$. Теорема 6.2 доказана.

Поскольку в рассуждениях вьше допустимо $h \equiv 0$, то одновременно обоснован нижний горизонтальньй переходв диаграммах (1.10) и (6.4). Значит, и теорема 6.1 доказана полностью.

\section{$\S 10$. О сходимости спектра}

Задачи (8.1) и (6.3) можно записать в операторной форме

$$
\mathscr{A}^{\varepsilon} u^{\varepsilon}=f^{\varepsilon}, \quad \mathscr{A} u=f,
$$

где $\mathscr{A}^{\varepsilon}=\mathscr{A}^{\varepsilon, h}, f^{\varepsilon}=f^{\varepsilon, h}$ при $h=h(\varepsilon), \mathscr{A}^{\varepsilon}, \mathscr{A}$ - неотрицательные самосопряженные операторы в гильбертовых пространствах $H_{\varepsilon}=L^{2}\left(\Omega, d \mu_{\varepsilon}^{h}\right)^{2}$ и $H=L^{2}(\Omega, d x)^{2}$ соответственно, $\mathscr{A}^{\varepsilon}$ и $\mathscr{A}$ имеют компактную резольвенту.

Распространим обычные теоремы о спектральных асимптотиках, вытекающие из сильной резольвентной сходимости [23], [18], на случай переменных гильбертовых пространств.

Рассмотрим обшую схему, в которой нам даны только сильная резольвентная сходимость в переменном гильбертовом пространстве и абстрактньй принцип компактности.

Для ограниченных операторов $B_{\varepsilon}, B$, действующих в пространствах $H_{\varepsilon}$ и $H$ соответственно, таких, что $\lim \sup _{\varepsilon \rightarrow 0}\left\|B_{\varepsilon}\right\|<\infty$, будем рассматривать сильную сходимость $B_{\varepsilon} \rightarrow B$, которая означает, что $B_{\varepsilon} u_{\varepsilon} \rightarrow B u$ в $H_{\varepsilon}$, как только $u_{\varepsilon} \rightarrow u$ в $H_{\varepsilon}$. Здесь мы оперируем некоторой "сильной" сходимостью $u_{\varepsilon} \rightarrow u$ элементов $u_{\varepsilon} \in H_{\varepsilon}$ к элементу $u \in H$, обладающей следующими свойствами.

$1^{\circ}$. Если $u_{\varepsilon} \rightarrow u, v_{\varepsilon} \rightarrow v$, то $\left(u_{\varepsilon}, v_{\varepsilon}\right)_{\varepsilon} \rightarrow(u, v)$.

$2^{\circ}$. Для любого $u \in H$ найдется $u_{\varepsilon} \in H_{\varepsilon}$ такая, что $u_{\varepsilon} \rightarrow u$. 
Введем условия:

$\left(\Gamma_{1}\right)\left(\mathscr{A}^{\varepsilon}+\lambda\right)^{-1} \rightarrow(\mathscr{A}+\lambda)^{-1} \forall \lambda>0 ;$

$\left(\Gamma_{2}\right)$ в пространстве $H_{\varepsilon}$ имеет место принцип компактности, а именно:

$$
\text { если } \limsup _{\varepsilon \rightarrow 0}\left(\left(\mathscr{A}^{\varepsilon}+1\right) u_{\varepsilon}, u_{\varepsilon}\right)_{\varepsilon}<\infty, \quad \text { то } \exists \varepsilon^{\prime}: u_{\varepsilon^{\prime}} \rightarrow u .
$$

Из $\left(\Gamma_{1}\right)$ следует простой факт, который используется далее:

$$
\text { если } \mathscr{A}_{\varepsilon} u_{\varepsilon}-\lambda u_{\varepsilon}=o(1) \text { в } H_{\varepsilon}, u_{\varepsilon} \rightarrow u \text {, то } \mathscr{A} u=\lambda u \text {. }
$$

Теорема 10.1. Из условий $\left(\Gamma_{1}\right)$ и $\left(\Gamma_{2}\right)$ следует сходимость по Хаусдорфу $\operatorname{Sp} \mathscr{A}^{\varepsilon} \rightarrow \operatorname{Sp} \mathscr{A}:$

$\left(\mathrm{H}_{1}\right) \forall \lambda \in \operatorname{Sp} \mathscr{A} \quad \exists \lambda_{\varepsilon} \in \mathrm{Sp} \mathscr{A}^{\varepsilon}$ maкое, ито $\lambda_{\varepsilon} \rightarrow \lambda ;$

$\left(\mathrm{H}_{2}\right)$ ecлu $\lambda_{\varepsilon} \in \mathrm{Sp} \mathscr{A}^{\varepsilon}, \lambda_{\varepsilon} \rightarrow \lambda, \lambda<\infty$, mo $\lambda \in \mathrm{Sp} \mathscr{A}$.

ДокАЗАТЕЛЬСТво. Свойство $\left(\mathrm{H}_{1}\right)$ следует только из условия $\left(\Gamma_{1}\right)$, как это показано в [24]. Докажем здесь свойство $\left(\mathrm{H}_{2}\right)$. Для $\lambda_{\varepsilon} \in \mathrm{Sp} \mathscr{A}^{\varepsilon}$ найдутся

$$
u_{\varepsilon} \in H_{\varepsilon}, \quad \mathscr{A}^{\varepsilon} u_{\varepsilon}-\lambda_{\varepsilon} u_{\varepsilon}=o(1) \text { в } H_{\varepsilon}, \quad\left(u^{\varepsilon}, u^{\varepsilon}\right)_{\varepsilon}=1 .
$$

Можно считать, что $u_{\varepsilon} \rightarrow u$ (следует из $\left(\Gamma_{2}\right)$ ). Переход к пределу дает соотношения

$$
\mathscr{A} u=\lambda u, \quad(u, u)=1,
$$

из которых следует, что $\lambda \in \operatorname{Sp} \mathscr{A}$. Теорема доказана.

Пусть $\mathscr{A}^{\varepsilon}=\int_{0}^{\infty} \lambda d E_{\varepsilon}(\lambda), \mathscr{A}=\int_{0}^{\infty} \lambda d E(\lambda)$ - спектральные разложения операторов $\mathscr{A}^{\varepsilon}, \mathscr{A}$.

Tеорема 10.2. В предположсении $\left(\Gamma_{1}\right)$, если $\lambda$ не есть собственное значение $\mathscr{A}$, mо

$$
E_{\varepsilon}(\lambda) \rightarrow E(\lambda) .
$$

Эта теорема доказана в работе [24].

Теорема 10.3. В предположении $\left(\Gamma_{1}\right)$, если $\lambda$ не есть собственное значение $\mathscr{A}$, mо

$$
\operatorname{dim} E_{\varepsilon}(\lambda) H_{\varepsilon} \geqslant \operatorname{dim} E(\lambda) H \quad \forall \varepsilon<\varepsilon_{0} .
$$

ДоКАЗАТЕЛЬСтво. Пусть $u_{1}, \ldots, u_{n}$ - ортонормированная система в $E(\lambda) H$. Для любого $i$ сушествует $\widehat{u}_{i}^{\varepsilon} \in H_{\varepsilon}$ такой, что $\widehat{u}_{i}^{\varepsilon} \rightarrow u_{i}$. Пусть $u_{i}^{\varepsilon}=E_{\varepsilon}(\lambda) \widehat{u}_{i}^{\varepsilon}$, тогда по теореме 10.2

$$
\left(u_{i}^{\varepsilon}, u_{j}^{\varepsilon}\right)_{\varepsilon}=\left(E_{\varepsilon}(\lambda) \widehat{u}_{i}^{\varepsilon}, \widehat{u}_{j}^{\varepsilon}\right)_{\varepsilon} \rightarrow\left(E(\lambda) u_{i}, u_{j}\right)=\left(u_{i}, u_{j}\right)=\delta_{i j}
$$

и, значит, матрища Грама векторов $u_{1}^{\varepsilon}, \ldots, u_{n}^{\varepsilon}$ невырождена при $\varepsilon$ достаточно малых, так как

$$
\operatorname{det}\left\|\left(u_{i}^{\varepsilon}, u_{j}^{\varepsilon}\right)_{\varepsilon}\right\|_{i, j=1}^{n} \rightarrow 1 \text {. }
$$

Следовательно, $u_{i}^{\varepsilon}, \ldots, u_{n}^{\varepsilon}-$ линейно независимы и неравенство (10.1) вьполнено. Теорема доказана. 
ТЕОРема 10.4. В предположении $\left(\Gamma_{1}\right),\left(\Gamma_{2}\right)$, если $\lambda$ не является собственным значением $\mathscr{A}$, то

$$
\operatorname{dim} E_{\varepsilon}(\lambda) H_{\varepsilon} \leqslant \operatorname{dim} E(\lambda) H \quad \forall \varepsilon<\varepsilon_{0} .
$$

ДокАЗАТЕЛЬСтво. Взяв произвольное $\lambda$, считаем, что

$$
\operatorname{dim} E(\lambda) H=n<\infty
$$

в случае $\operatorname{dim} E(\lambda) H=\infty$ доказывать нечего. Предположив, что (10.2) неверно, найдем подпоследовательность $\varepsilon^{\prime} \subset \varepsilon$ такую, что при $\varepsilon=\varepsilon^{\prime} \rightarrow 0$

$$
\operatorname{dim} E_{\varepsilon}(\lambda) H_{\varepsilon}>n
$$

Сначала для ясности допустим, что операторы $\mathscr{A}_{\varepsilon}$ имеют компактные резольвенты. Тогда найдутся векторы $u_{1}^{\varepsilon}, \ldots, u_{n+1}^{\varepsilon}$ такие, что

$$
\mathscr{A}^{\varepsilon} u_{i}^{\varepsilon}=\lambda_{i}^{\varepsilon} u_{i}^{\varepsilon}, \quad\left(u_{i}^{\varepsilon}, u_{j}^{\varepsilon}\right)_{\varepsilon}=\delta_{i j}, \quad i=1,2, \ldots, n+1, \quad \lambda_{i}^{\varepsilon} \leqslant \lambda .
$$

Как и при доказательстве теоремы 10.1, предельный переход дает

$$
\mathscr{A} u_{i}=\lambda_{i} u_{i}, \quad\left(u_{i}, u_{j}\right)=\delta_{i j}, \quad i, j=1,2, \ldots, n+1, \quad \lambda_{i} \leqslant \lambda,
$$

что противоречит (10.3). Если резольвенты операторов $\mathscr{A}_{\varepsilon}$ не являются компактными, в (10.4) надо взять “почти собственные” векторы. Теорема доказана.

Аналогично устанавливается такое утверждение: если $\lambda_{1}<\lambda_{2}$ не являются собственными значениями оператора $\mathscr{A}$, то

$$
\lim _{\varepsilon \rightarrow 0} \operatorname{dim}\left(E\left(\lambda_{2}\right)-E\left(\lambda_{1}\right)\right) H_{\varepsilon}=\operatorname{dim}\left(E\left(\lambda_{2}\right)-E\left(\lambda_{1}\right)\right) H .
$$

В свою очередь, отсюда получаем следующую теорему.

Tеорема 10.5. Пусть $\lambda$ - собственное значение кратности $r$ оператора $\mathscr{A}$ и в интервале $I=(-\delta+\lambda, \lambda+\delta)$ нет других собственных значений оператора А. Тогда для достаточно малых в в интервале I содержится ровно $r$ собственных значений оператора $\mathscr{A}^{\varepsilon}$ с учетом их кратности.

Теперь введем новые предположения. Сначала допустим, что предельный оператор $\mathscr{A}$ имеет компактную резольвенту. При этом оператор $\mathscr{A}^{\varepsilon}$ может иметь непрерывный спектр. Из теорем $10.1,10.5$ следует, что при малых $\varepsilon$ оператор $\mathscr{A}^{\varepsilon}$ обязательно имеет собственные значения (вблизи собственных значений оператора $\mathscr{A}$ и той же кратности), а непрерывный спектр “уходит на бесконечность" при $\varepsilon \rightarrow 0$. Другими словами, спектр оператора $\mathscr{A}^{\varepsilon}$ "асимптотически" дискретен.

Наконец, допустим, что и оператор $\mathscr{A}^{\varepsilon}$ имеет компактную резольвенту. Упорядочим собственные значения по принципу минимакса и пусть

$$
\begin{gathered}
\left\{u_{i}^{\varepsilon}\right\}-\text { ортогональный базис в } H_{\varepsilon}, \quad \mathscr{A}^{\varepsilon} u_{i}^{\varepsilon}=\lambda_{\varepsilon}^{i} u_{i}^{\varepsilon}, \\
\left\{u_{i}\right\}-\text { ортогональный базис в } H, \quad \mathscr{A} u_{i}=\lambda^{i} u_{i} .
\end{gathered}
$$

Пусть $K(\lambda)$ - подпространство собственных векторов с собственным значением $\lambda \in \operatorname{Sp}(\mathscr{A})$. 
ТЕОРЕМа 10.6. Собственные значения и собственные векторы операторов $\mathscr{A}^{\varepsilon}$ и $\mathscr{A}$ связаны соотношениями:

(i) $\lambda_{k}^{\varepsilon} \rightarrow \lambda_{k}$ npu $\varepsilon \rightarrow 0, k=1,2, \ldots$;

(ii) $\exists \varepsilon^{\prime} \subset \varepsilon: u_{k}^{\varepsilon^{\prime}} \rightarrow U_{k} \in K\left(\lambda_{k}\right),\left\|U_{k}\right\|=1, k=1,2, \ldots$;

(iii) $u_{k}^{\varepsilon} \rightarrow u_{k}$, если $\lambda_{k}$ - некратное собственное значение.

ДокаЗАтЕльство. Пусть для данного $k \widehat{\lambda}_{1}<\widehat{\lambda}_{2}<\cdots<\widehat{\lambda}_{j}=\lambda_{k}-$ все различные собственные значения оператора $\mathscr{A}$, не превосходящих $\lambda_{k}$, при этом $r_{i}$ - кратность $\widehat{\lambda}_{i}, r_{1}+\cdots+r_{j}=m \geqslant k$. Выберем непересекающиеся интервалы $I_{1}, \ldots, I_{j}$, содержащие по одной точке $\widehat{\lambda}_{i}$. По теореме 10.4 при достаточно малых $\varepsilon$ $m$ первых собственных значений $\lambda_{1}^{\varepsilon}, \ldots, \lambda_{m}^{\varepsilon}$ попадают последовательно в интервалы $I_{1}, \ldots, I_{j}$ в соответствии с кратностью $r_{1}, \ldots, r_{j}$. Стягивая интервал $I_{i}$ к точке $\widehat{\lambda}_{i}$, получаем сходимость $\lambda_{i}^{\varepsilon} \rightarrow \lambda_{i}, i=1, \ldots, m$. В частности, $\lambda_{k}^{\varepsilon} \rightarrow \lambda_{k}$. Утверждение $(\mathrm{i})$ доказано. Из него в силу $\left(\Gamma_{1}\right),\left(\Gamma_{2}\right)$ следует (ii).

Рассмотрим случай некратного $\lambda_{k}$. Тогда для подпоследовательности $\varepsilon^{\prime}$ из п. (ii) $U_{k}= \pm u_{k}$, так как $\left\|U_{k}\right\|=\left\|u_{k}\right\|=1$, $\operatorname{dim} K\left(\lambda_{k}\right)=1$. Найдется последовательность $U_{k}^{\varepsilon}, U_{k}^{\varepsilon} \rightarrow U_{k}$, для которой $\left(u_{k}^{\varepsilon^{\prime}}, U_{k}^{\varepsilon^{\prime}}\right) \varepsilon_{\varepsilon^{\prime}} \geqslant 0$. Тогда в пределе $\left(u_{k}, U_{k}\right) \geqslant 0$ и, значит, $U_{k}=u_{k}$. Отсюда следует утверждение (iii). Теорема доказана.

\section{§ 11. Об аппроксимативных условиях}

1. Пусть $\mu^{h}$ - периодическая нормированная борелева мера в $\mathbb{R}^{d}$ с ячейкой периодичности $Y=[-1 / 2,1 / 2)^{d}$, слабо сходящаяся к мере $\mu: \mu^{h} \rightarrow \mu$. Здесь нам удобно рассматривать соболевские пространства теории упругости как множества пар $(u, v)$, состоящих из вектора $u$ и его градиента $v=e(u)$. Например, $W_{\text {per }}=W_{\text {per }}(Y, d \mu)$ есть замькание множества $\left\{(\varphi, e(\varphi)), \varphi \in C_{\text {per }}^{\infty}(Y)^{d}\right\}$ в $L^{2}(Y, d \mu)^{d} \times L^{2}(Y, d \mu)^{d(d+1) / 2}$. Привычнее называть соболевским пространство $\mathscr{H}_{\text {per }}=\left\{u:(u, v) \in W_{\text {per }}\right\}$.

Для меры $\mu^{h}$ введем аппроксимативные условия (или свойства) так же, как для составной меры в $\S 5$, см. определение 5.3 .

ОПРЕДЕЛЕНИЕ 11.1. Скажем, что в пространстве $\mathscr{H}_{\mathrm{per}}\left(Y, \mu^{h}\right)$ возможен предельный переход, если

$$
(u, v) \in W_{\text {per }}(Y, d \mu), \quad \text { т.е. } v=e(u)
$$

как только

$$
u^{h} \rightarrow u, \quad e\left(u^{h}\right) \rightarrow v \quad \text { в } L^{2}\left(Y, d \mu^{h}\right)
$$

Оказывается, что аппроксимативные условия и возможность предельного перехода тесно связаны. Чтобы убедиться в этом, удобно оперировать двойственным определением соболевского пространства теории упругости (для пространства скалярных функций это определение введено В. В. Жиковьм [25]). Будем использовать отношение, определенное в случае составной меры в $\S 5$ :

$$
a=\operatorname{div} b \quad(\text { по мере } \mu),
$$

между вектором $a$ и симметрической матрицей $b$ из $L^{2}(Y, d \mu)$. 
ОПРЕДЕЛЕНИЕ 11.2. Скажем, что пара $(u, v)$, где

$$
u \in L^{2}(Y, d \mu)^{d}, \quad v \in L^{2}(Y, d \mu)^{d(d+1) / 2}
$$

принадлежит пространству $\widetilde{W}_{\text {per }}$, если

$$
\int_{Y} a \cdot u d \mu=-\int_{Y} b \cdot v d \mu
$$

как только $a=\operatorname{div} b($ по мере $\mu$ ).

Непосредственно из определений выводятся следующие два утверждения.

Tеорема 11.3. Пространства $W_{\text {per }}$ и $\widetilde{W}_{\text {per }}$ совпадают.

ТЕОРЕМА 11.4. Из аппроксимативного условия (i) следует возможсность предельного перехода в $\mathscr{H}_{\mathrm{per}}\left(Y, d \mu^{h}\right)$.

Предположим, что для меры $\mu^{h}$ пространство жестких периодических перемещений состоит только из постоянных векторов, т.е.

$$
\mathscr{R}\left(\mu^{h}\right)=\mathbb{R}^{d}
$$

ТЕОРема 11.5. Пусть в дополнение к условию (11.4) выполнено равномерное по $һ$ неравенство Корна (7.22). Тогда возмохнность предельного перехода в соболевском пространстве $\mathscr{H}_{\mathrm{per}}\left(Y, d \mu^{h}\right)$ влечет выполнение аппроксимативных условий (i), (ii).

ДокАЗАТЕЛЬСТво. Для $a$ и $b$, удовлетворяюших отношению (11.3), возьмем какие-то апшроксимации $\widetilde{a}^{h} \rightarrow a, \widetilde{b}^{h} \rightarrow b$ в $L^{2}\left(Y, d \mu^{h}\right)$. Для $a^{h}=\widetilde{a}^{h}-\int_{Y} \widetilde{a}^{h} d \mu^{h}$ по-прежнему имеем сходимость $a^{h} \rightarrow a$ в $L^{2}\left(Y, d \mu^{h}\right)$. Рассмотрим периодическую задачу

$$
u^{h} \in \mathscr{H}_{\text {per }}\left(Y, d \mu^{h}\right), \quad \operatorname{div} e\left(u^{h}\right)=\operatorname{div} \widetilde{b}^{h}-a^{h}, \quad \int_{Y} u^{h} d \mu^{h}=0,
$$

решение которой удовлетворяет интегральному тождеству

$$
\int_{Y} e\left(u^{h}\right) \cdot e(\varphi) d \mu^{h}=\int_{Y} \widetilde{b}^{h} \cdot e(\varphi) d \mu^{h}+\int_{Y} a^{h} \cdot \varphi d \mu^{h} .
$$

Сушествование решения и равномерная ограниченность $u^{h}, e\left(u^{h}\right)$ в $L^{2}\left(Y, d \mu^{h}\right)$ следуют из неравенства Корна (7.22). Поэтому можно считать, что имеют место сходимости (11.2) и, значит, по предположению выполнены соотношения (11.1). Взяв в тождестве (11.5) $\varphi=u^{h}$, после перехода к пределу с учетом сходимостей для $a^{h}$ и $\widetilde{b}^{h}$ получаем

$$
\lim _{h \rightarrow 0} \int_{Y} e\left(u^{h}\right) \cdot e\left(u^{h}\right) d \mu^{h}=\int_{Y}[b \cdot e(u)+a \cdot u] d \mu=0
$$

в силу (11.3) и определения 11.2 , т.е. $e\left(u^{h}\right) \rightarrow 0$ в $L^{2}\left(Y, d \mu^{h}\right)$.

Полагая $b^{h}=\widetilde{b}^{h}-e\left(u^{h}\right)$, получаем искомую сильную аппроксимацию матрицы $b$ и вместе с тем аппроксимативное свойство (i). Одновременно проверено и свойство (ii): для $a \equiv 0$ берем $a^{h} \equiv 0$. Теорема доказана. 
2. Вернемся к составным мерам. Пусть $F$ - произвольньй составленный из отрезков 1-периодический связный граф на плоскости (сетка), $Y=[-1 / 2,1 / 2)^{2}-$ ячейка периодичности. Заменяя каждое звено $I$ из $F$ на полосу, ширина которой равна $h$, а средняя линия совпадает с $I$, получаем тонкую сетку $F^{h}$. Пусть $\lambda, \lambda^{h}$ - естественные меры на сетках $F, F^{h}$ и $\mu, \mu^{h}$ - соответствующие составные меры, см. (1.1).

Будем предполагать, что в каждой вершине графа $F$ сходятся хотя бы два ребpa. В этом случае можно дать описание пространства $\mathscr{H}_{\mathrm{per}}(Y, d \mu)$ и проверить аппроксимативные условия.

Tеорема 11.6. Имеет место следующее: $u \in \mathscr{H}_{\mathrm{per}}(Y, d \mu) \Leftrightarrow$

(i) $u \in H_{\mathrm{per}}^{1}(Y, d \mu)^{2}$;

(ii) $\left.u\right|_{F} \in \mathscr{H}_{\mathrm{per}}(Y, d \lambda)$, где $\left.u\right|_{F}-$ след функции $и \in H_{\mathrm{per}}^{1}(Y, d \mu)^{2}$ на $F$.

ТеОРема 11.7. Для составной меры $\mu^{h}$ выполнены аппроксимативные условия (i), (ii).

Для доказательства теоремы 11.7 достаточно (см. теорему 11.5 и неравенство Корна (9.1)) заметить возможность предельного перехода в $\mathscr{H}_{\text {per }}\left(Y, d \mu^{h}\right)$, которая вытекает (см. теорему 11.6) из возможности предельного перехода в пространствах $H_{\text {per }}^{1}(Y, d x)^{2}$ и $\mathscr{H}_{\text {per }}\left(Y, d \lambda^{h}\right)$. Этот факт для сеточного пространства вьводится (см. теорему 11.4) из аппроксимативных свойств меры $\lambda^{h}$, проверенных в [26], или может быть доказан непосредственно (см. [27]).

Настояшая работа представляет собой расширенный вариант статьи [28].

\section{Список литературы}

1. Жиков В. В. К технике усреднения вариационных задач // Функц. анализ и его прилож. 1999. Т. 33. № 1. C. 14-29.

2. Жиков В. В. Об одном расширении и применении метода двухмасштабной сходимости // Матем. сб. 2000. Т. 191. № 7. С. 31-72.

3. Жиков В. В. Усреднение задач теории упругости на сингулярных структурах // Изв. РАН. Сер. матем. 2002. Т. 66. № 2. С. 81-148.

4. Жиков В. В. Усреднение задач теории упругости на сингулярных структурах // Докл. PAH. 2001. T. 380. №6. C. 741-745.

5. Жиков B. В., Пастухова C. Е. Усреднение задач теории упругости на периодических сетках критической толщины // Матем. сб. 2003. Т. 194. № 5. С. 61-95.

6. Жиков В. В., Пастухова C. Е. О неравенствах Корна на тонких периодических структурах // Докл. РАН. 2003. Т. 388. № 5. С. 588-592.

7. Bouchitté G., Fragalà I. Homogenization of thin structures by two-scale method with respect to measures // SIAM J. Math. Anal. 2001. V. 32 (6). P. 1198-1226.

8. Chiadò Piat V., Zhikov V. V. Relaxation problems in Sobolev spaces with respect to a measure // Rapporto interno. № 18, luglio 2003, Politecnico di Torino.

9. Жиков В. В., Пастухова С. Е. Об усреднении на периодических сетках // Докл. РАН. 2003. T. 391. № 4. C. 443-447.

10. Жиков В. В., Пастухова C.E. Об усредненном тензоре на периодических сетках // Труды МИАН (в печати).

11. Бахвалов Н. С., Панасенко Г. П. Осреднение процессов в периодических средах. М.: Наука, 1984.

12. Панасенко Г. П. Осреднение периодических структур с хорошо проводящими неоднородностями // Вестн. МГУ. Сер. вычислительная математика и кибернетика. 1980. Т. 3. C. $4-11$. 
13. Панасенко Г. П. Осреднение полей в композитных материалах с высоко модульной арматурой // Вестн. МГУ. Сер. вычислительная математика и кибернетика. 1983. Т. 2. C. $20-27$.

14. Косарев А. Ю. Асимптотика осредненных характеристик периодических упругих сред с сильно изменяющимися свойствами // Докл. АН СССР. 1982. Т. 267. № 1. С. 38-42.

15. Сандраков Г. В. Осреднение системы уравнений теории упругости с контрастньми коэффицциентами // Матем. сб. 1999. Т. 190. № 12. С. 37-92.

16. Санчес-Паленсия Э. Неоднородные среды и теория колебаний. М.: Мир, 1984.

17. Sanchez-Palencia E. Problèmes de perturbations lies aux phènoménes de conduction à travers des couches minces de grande résistivité // J. Math. Pures Appl. (9). 1974. V. 53. P. 251-270.

18. Mosco U. Compositemedia and asymptotic Dirichlet forms // J. Funct. Anal. 1994. V. 123. P. $368-421$.

19. Жиков В. В. О весовых соболевских пространствах // Матем. сб. 1998. Т. 189. №8. C. $27-58$.

20. Жиков В. В. О двухмасштабной сходимости // Труды сем. имени И.Г. Петровского. 2003. T. 23. C. $149-187$.

21. Nguetseng G. A general convergence result for a functional related to the theory of homogenization // SIAM J. Math. Anal. 1989. V. 20. P. 608-623.

22. Allaire G. Homogenization and two-scale convergence // SIAMJ. Math. Anal. 1992. V. 23. P. $1482-1518$.

23. Като Т. Теория возмущений линейных операторов. М.: Мир, 1972.

24. Пастухова C. E. О сходимости гиперболических полугрупп в переменном гильбертовом пространстве // Труды сем. имени И. Г. Петровского. 2004. Т. 24. С. 216-241.

25. Zhikov V. V. Note on Sobolev space // Contemp. Math. 2003. V. 10. P. 54-58.

26. Пастухова C. E. Об аппроксимативных свойствах соболевских пространств теории упругости на тонких стержневых структурах // Современная матем. и ее прилож. 2003. T. 12. C. 99-106.

27. Пастухова $C$. E. Аппроксимативные условия и предельный переход в соболевских пространствах на тонких и составных структурах // Современная матем. и ее прилож. 2004. T. 16. C. $47-63$.

28. Пастухова C.E. Усреднение задач теории упругости на периодических составных структурах // Докл. РАН. 2004. Т. 395. №3. С. 316-321.

Московский институт радиотехники, электроники и автоматики (технический университет)

Поступила в редакцию 07.10 .2003 и 13.09 .2004 